\title{
Topological groups of Kac-Moody type, right-angled twinnings and their lattices
}

\author{
Bertrand Rémy and Mark Ronan
}

\begin{abstract}
We construct new groups satisfying the combinatorial axioms of twin root data, by amalgamating spherical parabolic subgroups. The corresponding buildings have right-angled Coxeter groups as Weyl groups. The possibility of mixing several ground fields, not available for Kac-Moody groups, leads to strong non-linearity properties for the groups and some of their subgroups. We also discuss a completion procedure for groups with twin root data which is the starting point for defining a large family of topologically simple groups.
\end{abstract}

Mathematics Subject Classification (2000). 20E42, 51E24, 20G25, 22E40, 17 B67.

Keywords. Twin building, twin root datum, tree, right-angled building, lattice, non-linearity.

\section{Introduction}

In this paper we construct groups acting on right-angled buildings. Our groups are generated by root groups in a way that places a Moufang structure on the building. This yields a twin building which in turn leads to lattices acting on the original building. As a special case we obtain non-uniform lattices acting on right-angled hyperbolic buildings.

A building is called right-angled if its Coxeter system $(W, S)$ is right-angled, where $W$ denotes the Coxeter group, and $S$ a canonical set of generators. This means that the order of the product of any two generators $s_{i}$ and $s_{j}$ in $S$ is either 2 or infinity. For such buildings each rank 2 residue is either a complete bipartite graph or a tree. There is no loss of generality in assuming that the Coxeter system is irreducible, and the rank is at least 2 , which implies that the building contains tree residues.

The property of being right-angled implies, in a suitable geometric realization, that the panels of each chamber are either at right angles or are disjoint. For example the hyperbolic plane tiled by right-angled $n$-gons is an apartment of such a type; the chambers are the $n$-gons, the panels are their edges, and buildings of this type have rank $n$ and are 2-dimensional hyperbolic. 
In order to state a theorem, let the valency of a panel denote the cardinality of the set of chambers on that panel. If all panels of type $s$ have the same valency $v(s)$ then we say the building has parameters $\{v(s)\}_{s \in S}$. Our construction in Section 3.E, based on our construction of Moufang trees in Section 2, yields the following theorem.

Theorem. Any right-angled building having a parameter system whose parameters are the cardinalities of projective lines admits a Moufang structure.

A Moufang structure is defined in terms of root group actions (see [Ro1] and [T5]). For the purposes of the present paper we lay out the axioms in terms of a "twin root datum" in Section 1.A. Such data immediately yield twin buildings having a Moufang structure. A twin building is a pair of buildings having the same Coxeter system $(W, S)$, along with a "codistance", taking values in $W$, between chambers of one building and chambers of the other. In a twin building the stabilizer of a face in one building acts on the other building. If $\Delta$ is a building that is Moufang and locally finite, a stabilizer in the building twinned with it acts as a lattice on $\Delta$ (if the parameters are sufficiently large). We should mention here that the geometric realization of our buildings uses only the spherical residues. When the parameters are finite this implies that the link of each vertex is finite.

Our construction uses an action of the group $\mathrm{SL}_{2}\left(\mathbb{K}_{S}\right)$ on the set of chambers having a common panel of type $s$. The action is the same as the action on the projective line over $\mathbb{K}_{S}$ (see the theorem above). We allow the possibility of mixing fields of different characteristics (for different $s$ in $S$ ), and this gives groups containing infinite subgroups of finite exponent, prime to one another. This provides our second result, given more precisely in Section 4.A.

Theorem. There exist groups having right-angled twin root data such that any Borel subgroup for one building is a non-uniform lattice for the other building of the twin. Moreover any group homomorphism from such a lattice to a product of algebraic groups has infinite kernel.

These exotic groups share some combinatorial properties with algebraic and arithmetic groups, but provide non-classical applications of recent results generalizing properties of lattices in Lie groups [BS]. We can construct interesting non-linear topological groups for which well-known properties of harmonic analysis or unitary representations are relevant (and sometimes false, see the examples of the existence of a Gelfand pair or the Howe-Moore property considered in [BS, pp. 31-32]). This is done via a geometric completion procedure described in Section 1.B. This procedure is applied to groups having twin root data. For example, Kac-Moody groups admit twin root data (see [T2]), and when the group is over a finite field of characteristic $p$ our completion procedure leads to a topological Kac-Moody group having a refined Tits system in the sense of [KP] (see Section 1.A). In such cases the topological group 
is closer to an algebraic group over a local field than the non-linear topological groups mentioned above. More precisely we have the following pro- $p$-ness result (1.C).

Theorem. A topological Kac-Moody group admits a refined Tits system. The Tits system gives rise to a building in which any facet-fixator is, up to finite index, a pro- $p$ group.

This paper is organized as follows. In the first section, we introduce twin root data (1.A), define topological groups of root datum type (1.B), and show that they generalize algebraic groups over local fields (1.C). In Section 2 we sketch a construction of twin root data and apply it to trees; it is a special case of the construction of Moufang twin trees due to J. Tits, but given in a very down-to-earth way. The generators and relations that we provide in Section 2 are then used in Section 3 for our construction of right-angled buildings admitting root groups. Finally, in Section 4 we discuss linearity properties for lattices of topological groups of root datum type.

Let us state a convention we use for group actions. If a group $G$ acts on a set $X$, the pointwise stabilizer of a subset $Y \subset X$ is called its fixator and is denoted by $\operatorname{Fix}_{G}(Y)$. The global stabilizer is denoted by $\operatorname{Stab}_{G}(Y)$. When $Y$ is a facet of a building $X$ and $G$ is type-preserving, the two notions coincide. Finally the notation $\left.G\right|_{X}$ means the group obtained from $G$ by factoring out the kernel of the action on $X$.

The first author thanks University College, London where this work was initiated and the Hebrew University, Jerusalem for its warm hospitality (2000/2001). He was supported by grants from the British EPSRC and the Hebrew University. He thanks M. Bourdon for helpful discussions, Sh. Mozes for suggesting a stronger version of the non-linearity property, and J. Dymara and G. Rousseau for suggesting many improvements and pointing out mistakes. The second author was partially supported by the National Security Agency. We would also like to thank the referees for suggesting that the scope of the paper be widened from right-angled Fuchsian buildings to all right-angled buildings.

\section{Closures of groups with twin root data}

We introduce a family of totally disconnected topological groups, called topological groups of root datum type (1.B). This requires some basic properties of group combinatorics introduced by J. Tits and by V. Kac and D. Peterson (1.A). In the case of topological Kac-Moody groups, some precise structure results are available, leading to analogies with algebraic groups over local fields of characteristic $p$ (1.C).

1.A. Group combinatorics ([A], [Cho], [Hée], [KP], [Ro1], [Ro2], [T2], [T3], [T4], [T5]). Let $(W, S)$ be the Coxeter system $W=\langle s \in S|(s t)^{M_{s t}}=1$ whenever $\left.M_{s t}<\infty\right)$, where $M$ is a Coxeter matrix. The Coxeter complex $\Sigma=\Sigma(W, S)$ is 
the union of the translates $w W_{J}=w\langle J\rangle$ for $J \subset S$ and $w \in W$, partially ordered by reverse inclusion [Ro1, §2]. It is an abstract simplicial complex acted upon by $W$ on which are modelled apartments in buildings of type $(W, S)$. The elements $w=w\langle\varnothing\rangle$ have maximum dimension and are called chambers. The root system of $(W, S)$ is defined using the length function $\ell: W \rightarrow \mathbb{N}$ with respect to $S$ [T2, §5]: the simple root of index $s \in S$ is the subset $a_{s}=\{w \in W \mid \ell(s w)>\ell(w)\}$, and a root of $W$ is a translate $w a_{s}, w \in W, s \in S$. The set of all roots is denoted by $\Phi$; it admits an obvious $W$-action by left multiplication. A root is positive if it contains 1 ; otherwise, it is negative. We denote by $\Phi_{+}$(resp. $\Phi_{-}$) the set of positive (resp. negative) roots. The complement of a root $a$, denoted $-a$, is also a root, called the opposite of $a$. The wall $\partial a$ of a root $a$ is the union of the closed panels having exactly one chamber in $a$; it is also the intersection of the closures of $a$ and $-a$. A pair of roots $\{a ; b\}$ is called prenilpotent if both intersections $a \cap b$ and $(-a) \cap(-b)$ are nonempty. Given a prenilpotent pair of roots $\{a ; b\}$, the interval $[a ; b]$ is by definition the set of roots $c$ with $c \supset a \cap b$ and $(-c) \supset(-a) \cap(-b)$.

Definition. Let $\Lambda$ be an abstract group containing a subgroup $H$. Suppose $\Lambda$ is endowed with a family $\left\{U_{a}\right\}_{a \in \Phi}$ of subgroups, called root groups, indexed by the set of roots $\Phi$, and define the subgroups $U_{+}=\left\langle U_{a} \mid a \in \Phi_{+}\right\rangle$and $U_{-}=\left\langle U_{a} \mid a \in \Phi_{-}\right\rangle$. We say that the triple $\left(\Lambda,\left\{U_{a}\right\}_{a \in \Phi}, H\right)$ is a twin root datum for $\Lambda$ (or satisfies the (TRD) axioms) if the following conditions are fulfilled.

(TRD0) Each $U_{a}$ is nontrivial and normalized by $H$.

(TRD1) For each prenilpotent pair of roots $\{a ; b\}$, the commutator subgroup $\left[U_{a}, U_{b}\right]$ is contained in the subgroup $\left\langle U_{c}: c \in[a ; b] \backslash\{a ; b\}\right\rangle$.

(TRD2) For each $s \in S$ and $u \in U_{a_{s}} \backslash\{1\}$, there exist uniquely defined elements $u^{\prime}, u^{\prime \prime} \in U_{-a_{s}} \backslash\{1\}$ such that $m(u)=u^{\prime} u u^{\prime \prime}$ conjugates $U_{b}$ onto $U_{s . b}$ for every root $b$. Moreover, $m(u) H=m(v) H$ for all $u, v \in U_{a_{s}} \backslash\{1\}$.

(TRD3) For each $s \in S$, we have $U_{a_{s}} \not \subset U_{-}$and $U_{-a_{s}} \not \subset U_{+}$.

(TRD4) The group $\Lambda$ is generated by $H$ and the $U_{a}$ 's.

Examples. 1) The simplest example of a group with a twin root datum is $\mathrm{SL}_{2}$ over a field $k$. In the computational parts of Sections 2 and 3, we are led to handle several copies of it, so we use an index $i$ in describing certain elements and subgroups. The corresponding Coxeter group is $\mathbb{Z} / 2 \mathbb{Z}=\left\langle s_{i}\right\rangle$, so $\Phi=\left\{ \pm a_{i}\right\}$ and (TRD1) is an empty condition. We set $u_{i}(r)=\left(\begin{array}{ll}1 & r \\ 0 & 1\end{array}\right), u_{-i}(r)=\left(\begin{array}{cc}1 & 0 \\ -r & 1\end{array}\right), m_{i}(\lambda)=$ $u_{-i}\left(\lambda^{-1}\right) u_{i}(\lambda) u_{-i}\left(\lambda^{-1}\right), m_{i}=m_{i}(1)$ and $t_{i}(\lambda)=m_{i}(\lambda) m_{i}^{-1}$. The twin root datum is given by $U_{ \pm a_{i}}=u_{ \pm i}(k)$ and $H=t_{i}\left(k^{\times}\right)$. In matrix notation: $m_{i}(\lambda)=\left(\begin{array}{cc}0 & \lambda \\ -\lambda^{-1} & 0\end{array}\right)$ and $t_{i}(\lambda)=\left(\begin{array}{cc}\lambda & 0 \\ 0 & \lambda^{-1}\end{array}\right)$. Axiom (TRD3) is obvious and (TRD4) is well-known. Axiom (TRD0) follows from $t_{i}(\lambda) u_{ \pm i}(r) t_{i}(\lambda)^{-1}=u_{ \pm i}\left(\lambda^{ \pm 2} r\right)$, and finally (TRD2) follows from $m_{i}(\lambda) u_{i}(r) m_{i}(\lambda)^{-1}=u_{-i}\left(\lambda^{-2} r\right), m_{i}(\lambda) u_{-i}(r) m_{i}(\lambda)^{-1}=u_{i}\left(\lambda^{2} r\right)$ and 
$m_{i}(\lambda) m_{i}(\mu)=t_{i}\left(-\lambda \mu^{-1}\right)$. Note that we also have $m_{i}(\lambda) t_{i}(\mu) m_{i}(\lambda)^{-1}=t_{i}\left(\mu^{-1}\right)$, $m_{i}(\lambda)^{-1}=m_{i}(-\lambda)$ and $m_{i}^{2}=-$ id. Note that the uniqueness in (TRD2) follows from the rest of the axioms [T5, 3.3].

2) The special linear groups $\mathrm{SL}_{n}\left(k\left[t, t^{-1}\right]\right)$ over Laurent polynomials satisfy these axioms for $W$ affine of type $\widetilde{A}_{n-1}$. More generally, the groups $\mathbb{G}\left(k\left[t, t^{-1}\right]\right)$ where $\mathbb{G}$ is a simply connected Chevalley group, satisfy these axioms for a suitable affine Coxeter group $W([\mathrm{~A}, \S 1])$. In these cases, the root groups are naturally defined as subgroups of the spherical root groups by using polynomials of the form $a t^{r}$ for fixed $r$ as the off-diagonal entries. The commutator condition (TRD1) is carefully examined in [A, Examples 2 and 3].

The procedure to attach a building to a $B N$-pair $(G, B, N, S)$ is standard, see e.g. [Ro1, §5]: the simplices are in equivariant bijection with the translates $g P_{J}$ of the standard parabolic subgroups $P_{J}=B\langle J\rangle B, J \subset S$. The (TRD) axioms imply the existence of two $B N$-pairs in $G$ ([A, Proposition 1]), hence of two buildings. Moreover there is a natural opposition relation on chambers of opposite signs such that two chambers are opposite if the intersection of their stabilizers is minimal. This is the generic position for two chambers, and the set of opposites for a given chamber is sometimes called a big cell, referring to a well-known terminology for algebraic groups. The root groups $U_{a}$ act as root groups on each building of the twinning. The buildings are therefore Moufang in the sense of Tits [T5, §4.3]. Geometrically this property implies that given a codimension one simplex $\Pi$ and a chamber $c$ containing it, there is a group $U_{a}$ of building automorphisms fixing $c$ and acting simple-transitively on the chambers containing $\Pi$ and different from $c$ ([Ro1, 6.4]).

Finally, we will also need group combinatorics defined by V. Kac and D. Peterson $[\mathrm{KP}, \S 3]$.

Definition. A refined Tits system for a group $G$ is a sixtuple $\left(G, N, U_{+}, U_{-}, H, S\right)$, where $N, U_{+}, U_{-}, H$ are subgroups satisfying the following:

(RT1) We have $G=\left\langle N, U_{+}\right\rangle, H \triangleleft N, H<N_{G}\left(U_{+}\right) \cap N_{G}\left(U_{-}\right), W=N / H$ and $(W, S)$ is a Coxeter system.

(RT2) For each $s \in S$, we set $U_{s}=U_{+} \cap s^{-1} U_{-} s$; and for any $w \in W$ and $s \in S$ we require:

(RT2a) $U_{s} \neq\{1\}$ and $s^{-1}\left(U_{s} \backslash\{1\}\right) s \subset U_{s} s H U_{s}$,

(RT2b) either $w^{-1} U_{s} w \subset U_{+}$or $w^{-1} U_{s} w \subset U_{-}$,

(RT2c) $U_{+}=U_{s}\left(U_{+} \cap s^{-1} U_{+} s\right)$.

(RT3) If $u_{-} \in U_{-}, u_{+} \in U_{+}$and $n \in N$ are such that $u_{-} n u_{+}=1$, then $u_{-}=$ $u_{+}=n=1$. 
1.B. Topological groups. Let $\Lambda$ be a group with twin root datum $\left(\Lambda,\left\{U_{a}\right\}_{a \in \Phi}, H\right)$. Until the end of Section 1, we assume that the Weyl group $W$ (hence $\Phi$ ) is infinite and that all root groups $U_{a}$ are finite. We denote by $\Delta$ the simply connected nonpositively curved metric realization, due to M. Davis [D] and G. Moussong [Mou], of the positive building of $\Lambda$ [A, Proposition 1 and Example 6]. We set $q_{\min }=$ $\min _{a \in \Phi}\left|U_{a}\right|\left(=\min _{s \in S}\left|U_{a_{s}}\right|\right)$ and $q_{\min }=\max _{a \in \Phi}\left|U_{a}\right|\left(=\max _{s \in S}\left|U_{a_{s}}\right|\right)$. Since only spherical facets appear in the Moussong-Davis realization, $\Delta$ is locally finite. Our main motivation in defining topological groups of root datum type is geometric group theory, in which groups are studied via their actions on suitable spaces. The following lemma shows that taking the quotient by the kernel of the $\Lambda$-action on $\Delta$ is harmless; it follows easily from [Ré1, 1.5.4] and refined Tits system arguments.

Lemma 1. The kernel $K$ of the $\Lambda$-action on $\Delta$ consists of the elements in $H$ centralizing all root groups. The groups $U_{ \pm}$embed in $\Lambda / K$, hence so do the root groups, and $\left(\Lambda / K,\left\{U_{a}\right\}_{a \in \Phi}, H / K\right)$ is again a twin root datum with the same associated Coxeter system and twin buildings.

When $\Lambda$ is a Kac-Moody group, the kernel $K$ is the center $Z(\Lambda)$ [Ré1, 9.6.2], which is finite when the ground field is. For $\mathrm{SL}_{n}\left(\mathbb{K}\left[t, t^{-1}\right]\right)$, the buildings of $\Lambda$ are Euclidean $\widetilde{A}_{n-1}$-buildings, and the kernel $K$ is the group $\mu_{n}\left(\mathbb{F}_{q}\right)$ of $n$-th roots of unity in $\mathbb{F}_{q}$.

Convention. Until the end of 1.B, $\Lambda$ denotes a group with twin root datum as above factored out by the kernel of its action on the building $\Delta$. This group has a twin root datum with finite root groups and infinite Weyl group.

Given an automorphism $g \in \operatorname{Aut}(\Delta)$ and a finite union $C$ of facets, $O_{C}(g)=$ $\left\{h \in \operatorname{Aut}(\Delta):\left.h\right|_{C}=\left.g\right|_{C}\right\}$ is by definition an open neighborhood of $g$ in $\operatorname{Aut}(\Delta)$. The group $\Lambda$ is not discrete on the single building $\Delta$ because each facet fixator is transitive on opposite facets in $\Delta_{-}$, of which there are infinitely many.

Definition. (i) We call the closure $G=\bar{\Lambda}^{\operatorname{Aut}(\Delta)}$ a topological group of root datum type. When $\Lambda$ is a Kac-Moody group over a finite field, we call $G$ a topological Kac-Moody group.

(ii) We call the fixator $\operatorname{Fix}_{G}(F)$ of a facet $F$ the parahoric subgroup of $G$ associated to $F$, and we denote it by $G_{F}$. We call a chamber fixator an Iwahori subgroup.

Remark. An automorphism of $\Delta$ lies in $G$ if and only if it coincides with an element of $\Lambda$ on any finite set of facets.

Example. Consider the image of $\mathrm{SL}_{n}\left(\mathbb{F}_{q}\left[t, t^{-1}\right]\right)$ acting on the Euclidean building corresponding to the discrete valuation ring with uniformizer $t$. The kernel of the 
action is $\mu_{n}\left(\mathbb{F}_{q}\right)$ and the "completion" $G$ is $\operatorname{SL}_{n}\left(\mathbb{F}_{q}((t))\right) / \mu_{n}\left(\mathbb{F}_{q}\right)$. When $n>2, G$ is close to $\operatorname{Aut}(\Delta)$, but when $n=2, \Delta$ is a tree and $G$ is far from being all of $\operatorname{Aut}(\Delta)$.

We now consider discrete subgroups of $G$, which by the above example are analogous to some arithmetic groups over function fields. Fix a chamber $c_{-}$in $\Delta_{-}$, and let $\Gamma$ denote its stabilizer. Recall that for $q_{\min } \gg 1$, the parabolic subgroups of a given sign $\varepsilon$ are lattices of the locally finite CAT(0)-building $\Delta_{-\varepsilon}$ (see [CG], [Ré2]). Since the group $\Lambda$ acts by type-preserving isometries on $\Delta$, the stabilizer of a facet $F$ in $\Lambda$ is also its fixator. We denote it by $\Lambda(F)$. Recall also that two subgroups $\Gamma$ and $\Gamma^{\prime}$ of a given group $G$ are commensurable if they share a finite index subgroup. The commensurator of $\Gamma$ in $G$ is the group $\operatorname{Comm}_{G}(\Gamma)=\left\{g \in G: \Gamma\right.$ and $g \Gamma g^{-1}$ are commensurable\}.

Lemma 2. (i) For any two negative facets $F$ and $F^{\prime}, \Lambda(F)$ and $\Lambda\left(F^{\prime}\right)$ are commensurable.

(ii) For any negative facet $F, \Lambda$ lies in the commensurator $\operatorname{Comm}_{\operatorname{Aut}(\Delta)}(\Lambda(F))$.

Proof. Let $F$ and $F^{\prime}$ be two negative facets. Since $\Delta_{-}$is locally finite, the $\Lambda(F)$ transforms of $F^{\prime}$ are in finite number; we denote them by $\gamma_{1} . F^{\prime}, \ldots \gamma_{m} . F^{\prime}$ for some $\gamma_{i} \in \Lambda(F)$. Therefore, given any $\gamma \in \Lambda(F)$ one has $\gamma \cdot F^{\prime}=\gamma_{j} . F^{\prime}$ for some $j$, and hence $\Lambda(F)=\bigsqcup_{j=1}^{m} \gamma_{j}\left(\Lambda(F) \cap \Lambda\left(F^{\prime}\right)\right)$. Switching $F$ and $F^{\prime}$ proves (i), and (ii) follows from (i) since $g \Lambda(F) g^{-1}=\Lambda(g F)$.

Example. The negative vertex fixators in $\operatorname{SL}_{n}\left(\mathbb{F}_{q}\left(\left(t^{-1}\right)\right)\right) / \mu_{n}\left(\mathbb{F}_{q}\right)$ are conjugates of the subgroup $\operatorname{SL}_{n}\left(\mathbb{F}_{q}\left[\left[t^{-1}\right]\right]\right) / \mu_{n}\left(\mathbb{F}_{q}\right)$. In $\Lambda$ they are $\{0\}$-arithmetic groups commensurable with $\operatorname{SL}_{n}\left(\mathbb{F}_{q}\left[t^{-1}\right]\right) / \mu_{n}\left(\mathbb{F}_{q}\right)$, and the commensurator contains $\operatorname{SL}_{n}\left(\mathbb{F}_{q}(t)\right) / \mu_{n}\left(\mathbb{F}_{q}\right)$.

1.C. Kac-Moody groups. Let $\Lambda$ be the image in $\operatorname{Aut}(\Delta)$ of the rational points of an almost split Kac-Moody group over $\mathbb{F}_{q}$ [Ré1, 12.6.3] whose Weyl group is infinite. We denote its twin root datum by $\left(\Lambda,\left\{U_{a}\right\}_{a \in \Phi}, T\right)$, and let $G=\bar{\Lambda}^{\text {Aut }(\Delta)}$ denote the corresponding topological Kac-Moody group (1.B). By [Ré1, 12.5.4], for each root $a$ the group $U_{a}$ is isomorphic to the $\mathbb{F}_{q}$-points of the unipotent radical of a Borel subgroup in a rank one finite group of Lie type, so the finiteness of the root groups follows from that of $\mathbb{F}_{q}$, and $\left|U_{a}\right| \geq q$ for every $a \in \Phi$.

Example. The group $\mathrm{SU}_{3}\left(\mathbb{F}_{q}\left[t, t^{-1}\right]\right)$ is an almost split Kac-Moody group over $\mathbb{F}_{q}$ with infinite dihedral Weyl group. The associated buildings are semihomogeneous trees of valencies $1+q$ and $1+q^{3}$ [Ré4, 3.5].

The group $G$ admits a $B N$-pair, since its subgroup $\Lambda$ is strongly transitive (in the sense of $W$-distance) on $\Delta[$ Ro1, §5]. We now prove a stronger result stressing the 
analogy between $G$ and semisimple groups over local fields of positive characteristic. Let $F$ be a facet in $\Delta$ and let $\mathbb{A}$ be a twin apartment containing it [A, Lemma 2]. We have a Levi decomposition

$$
\Lambda(F)=M(\mathbb{A}, F) \ltimes U(F),
$$

where $M(\mathbb{A}, F)$ is abstractly isomorphic to the Kac-Moody group associated to a submatrix of the generalized Cartan matrix defining $\Lambda$ [Ré1, 6.2.2]. Since $F$ is spherical, $M(\mathbb{A}, F)$ is a finite reductive group of Lie type over $\mathbb{F}_{q}$. We denote by $U_{F}$ the closure $\overline{U(F)}{ }^{\operatorname{Aut}(\Delta)}$ of the unipotent radical $U(F)$.

Theorem. (i) The sixtuple $\left(G, N, U_{c}, U_{-}, T, S\right)$ defines a structure of refined Tits system for the topological Kac-Moody group $G$.

(ii) The group $U_{F}$ is pro- $p$ and the parahoric subgroup $G_{F}$ is virtually pro- $p$ since we have $G_{F}=M(\mathbb{A}, F) \ltimes U_{F}$.

Remark. The theorem implies that $\mathrm{SL}_{n}\left(\mathbb{F}_{q}((t))\right)$ satisfies the axioms of a refined Tits system, and that its parahoric subgroups admit semidirect product decompositions. This splitting of facet fixators is not true for algebraic groups over local fields of characteristic 0 . Other arguments, involving torsion, explain why the analogy with the characteristic 0 case is not relevant [Ré3].

To prove the theorem we need two lemmas which will be proved using a filtration $\left\{E_{n}\right\}_{n \geq 1}$ of $\Delta$. The first term $E_{1}$ is (the closure of) st $(F)$, the star of $F$ in $\Delta$ and further terms are defined inductively. Write $\mathbb{A}_{n}=E_{n} \cap \mathbb{A}$ and choose a chamber $c_{n}$ of $\mathbb{A}$ sharing a panel with the boundary of $\mathbb{A}_{n}$ and at minimal distance from $\mathbb{A}_{n}$. We define $\mathbb{A}_{n+1}$ to be the convex hull of $c_{n}$ and $\mathbb{A}_{n}$, and $E_{n+1}$ to be $\Lambda(F) . \mathbb{A}_{n+1}$, that is the set of all $\Lambda(F)$-transforms of $\mathbb{A}_{n+1}$. By the Bruhat decomposition, for any chamber $c \in \operatorname{st}(F), \mathbb{A}$ is a complete set of representatives for the action of $U(c)<\Lambda(F)$ on $\Delta$. Hence $\left\{E_{n}\right\}_{n \geq 1}$ exhausts $\Delta$. For each $n \geq 1$, the set $E_{n}$ is $\Lambda(F)$-stable. We can write the groups $G_{F}$ and $U_{F}$ as projective limits: $G_{F}=\left.\lim _{n \geq 1} \Lambda(F)\right|_{E_{n}}$ and $U_{F}=\left.\lim _{n \geq 1} U(F)\right|_{E_{n}}$.

Lemma 1. The group $U_{F}$ is pro-p.

Remark. This lemma is true for an arbitrary twin root datum as in 1.B, provided its root groups are $p$-groups.

Proof. For each $n \geq 1$, we have an exact sequence

$$
\left.\left.1 \longrightarrow K_{n} \longrightarrow U(F)\right|_{E_{n+1}} \longrightarrow U(F)\right|_{E_{n}} \longrightarrow 1,
$$

where $K_{n}$ is the fixator $\operatorname{Fix}_{\left.U(F)\right|_{n+1}}\left(E_{n}\right)$ of $E_{n}$ in the restricted group $\left.U(F)\right|_{E_{n+1}}$. 
Since $U(F)$ fixes st $(F)$, we have $\left.U(F)\right|_{E_{1}}=\{1\}$. By definition $U_{F}=\left.\lim _{n \geq 1} U(F)\right|_{E_{n}}$, so in order to show that $U_{F}$ is pro- $p$, it is enough by induction to show that $K_{n}$ is a $p$-group for each $n \geq 1$.

Let us fix $n \geq 1$ and $u \in K_{n}$. Let $\Pi_{n}$ be the panel of $c_{n}$ in the boundary of $\mathbb{A}_{n}$, and let $d_{n}$ be the other chamber of $\mathbb{A}$ having $\Pi_{n}$ as a panel. Then $d_{n} \in \mathbb{A}_{n}$, so $u$ fixes $d_{n}$, and by the Moufang property [Ro1, 6.4] there exists $v \in U_{a}$ such that $v^{-1} u \cdot c_{n}=c_{n}$. The element $v^{-1} u$ is in the "unipotent radical" of the Borel subgroup $\operatorname{Fix}_{\Lambda}\left(d_{n}\right)$ and fixes $c_{n} \cup d_{n}$; hence it must fix the whole star of $\Pi_{n}$. Consequently, $u$ and $v$ coincide on $\operatorname{st}\left(\Pi_{n}\right)$ and thus $u^{q_{\max }}$ acts trivially on it. In particular, $u^{q_{\max }}$ fixes $\mathbb{A}_{n} \cup\left\{c_{n}\right\}$ hence $\mathbb{A}_{n+1}$ by convexity. Every chamber of $E_{n+1} \backslash E_{n}$ is of the form $v . d$ for some $d$ in the convex hull of $\mathbb{A}_{n}$ and $c_{n}$, and $v \in \Lambda(F)$. Then $u^{q_{\max }}(v d)=v\left(v^{-1} u^{q_{\max }} v \cdot d\right)=$ $v\left(\left(v^{-1} u v\right)^{q_{\max }} . d\right)$. Recall that $U(F)$ is normalized by $\Lambda(F)$, so that applying the result of the above paragraph to $v^{-1} u v$ instead of $u$, we get $\left(v^{-1} u v\right)^{q_{\max }} \cdot d=d$, and thus $u^{q_{\max }}(v d)=v d$. This shows that the order of each element $u \in K_{n}$ divides $q_{\text {max }}$; hence $K_{n}$ is a $p$-group, as required.

Lemma 2. (i) We have $\Lambda \cap U_{F}=U(F)$, hence $M(\mathbb{A}, F) \cap U_{F}=\{1\}$ for any facet $F$. (ii) We have $G_{F}=M(\mathbb{A}, F) \ltimes U_{F}$.

Remark. In the proof below, the assumption that $\Lambda$ is Kac-Moody is used in asserting that the center of $M(\mathbb{A}, F)$ is an $\mathbb{F}_{q}$-torus, hence must intersect $U_{F}$ trivially.

Proof. (i) Let $g \in \Lambda \cap U_{F}<\operatorname{Fix}_{\Lambda}(F)=\Lambda(F)$. The Levi decomposition of $\Lambda(F)$ gives $g=m u$, with $m \in M(\mathbb{A}, F)$ and $u \in U(F)$. Since $U_{F}$ fixes st $(F)$, the set of chambers containing $F$, we see that both $g$ and $u$, hence also $m$, fix st $(F)$. Therefore $m$ is central in the finite reductive group $M(\mathbb{A}, F)$ of Lie type; this implies that $m$ lies in a torus, so its order is not divisible by $p$. But $m$ is also a torsion element in the pro- $p$ group $U_{F}$, and is hence trivial.

(ii) For each $n \geq 1, E_{n}$ is $\Lambda(F)$-stable by definition, hence $M(\mathbb{A}, F)$-stable. Since $M(\mathbb{A}, F)$ normalizes $U(F)$, it normalizes $\left.U(F)\right|_{E_{n}}$ in $\left.\Lambda(F)\right|_{E_{n}}$ for each $n \geq 1$. Hence $U_{F}$ is normalized by $M(\mathbb{A}, F)$. Let $g \in \Lambda(F)$. It can be written as $g=\lim _{n>1} g_{n}$, with $\left.g_{n} \in \Lambda(F)\right|_{E_{n}}$. According to the Levi decomposition of $\Lambda(F)$, we have $g_{n}=m_{n} u_{n}$ with $\left.m_{n} \in M(\mathbb{A}, F)\right|_{E_{n}}$ and $\left.u_{n} \in U(F)\right|_{E_{n}}$. The group $M(\mathbb{A}, F)$ is finite, so up to extracting a subsequence, we shall assume that $m_{n}$ is a constant element $m$ in $M(\mathbb{A}, F)$. This enables us to write $g=m \cdot\left(\lim _{n \geq 1} u_{n}\right)$, and proves $G_{F}=M(\mathbb{A}, F) \cdot U_{F}$. Finally (i) implies the trivial intersection $M(\mathbb{A}, F) \cap U_{F}=\{1\}$, hence the semidirect product decomposition $G_{F}=M(\mathbb{A}, F) \ltimes U_{F}$.

Proof of the theorem. By [Ré1, 1.5.4], $\left(\Lambda, N, U_{+}, U_{-}, T, S\right)$ is a refined Tits system with Weyl group $W$, and $U_{+} \cap s^{-1} U_{-} s=U_{a_{s}}$ for any $s \in S$. Axiom (RT1): the group $T$ normalizes $U(c)$, and passing to the projective limit it normalizes $U_{c}$. The other 
statements in axiom (RT1) are either clear or true from the refined Tits system axioms for $\Lambda$. Axiom (RT3): suppose we have $\gamma n u_{c}=1$, with $\gamma \in \Gamma, n \in N$ and $u_{c} \in U_{c}$. Then $\gamma n=u_{c}^{-1}$ belongs to $U_{c} \cap \Lambda$, which is $U(c)$ by Lemma 2: all the factors are in $\Lambda$ and we apply axiom (RT3) from the refined Tits system structure of $\Lambda$ to get (RT3) for $G$. Axioms (RT2): define $U(s)=U_{c} \cap s^{-1} \Gamma s$. The first part of Lemma 2 implies $U(s)<U(c) \cap s^{-1} \Gamma s$, hence $U(s)$ is the root group $U_{a_{s}}$ according to the relation of a refined Tits system and the twin root datum of a Kac-Moody group [Ré1, 1.6]. Axioms (RT2a) and (RT2b) are then clear because they just involve subgroups of $\Lambda$. For axiom (RT2c), we need $U_{c}=U_{a_{s}} \cdot\left(U_{c} \cap s^{-1} U_{c} s\right)$. An element $u \in U_{c}$ fixes $c$, hence permutes the chambers sharing their panel of type $s$ with $c$. Since $U_{a_{s}}$ fixes $c$ and is (simply) transitive on the set of chambers $\neq c$ of the latter type, there exists an element $v \in U_{a_{s}}$ such that $v^{-1} u$ fixes both $c$ and $s c$. This proves (RT2c).

The theorem gives an analogy between topological Kac-Moody groups and semisimple algebraic groups over local fields of positive characteristic. In the next two sections, we construct groups exotic enough to show that the analogy is strictly weaker for arbitrary twin root data.

\section{Twinning trees}

The construction of this section applies to trees as well as to right-angled buildings. Here are its main steps.

1. Define the "Borel subgroup" by means of a presentation described by an apartment.

2. To each type of spherical facets associate a "unipotent radical" and a "Levi factor".

3. Show that the "Levi factor" admits an action on the "unipotent radical".

4. Amalgamate these groups along the inclusions given by the closure of a chamber.

5. Check the twin root datum axioms for the amalgam $\Lambda$.

Step 4 amounts to taking the fundamental group of a suitable complex of groups. The computations for Step 3 are done in detail in the present section. This enables us to concentrate on more conceptual arguments in the case of right-angled buildings (Section 3).

2.A. Root system. The Coxeter complex here is a tiling of $\mathbb{R}$ by segments with vertices the integers. We denote by $E$ the segment $[0,1]$, and by $s_{0}$ (resp. $s_{1}$ ) the reflection with respect to 0 (resp. 1). This is the Coxeter complex of the infinite dihedral group $D_{\infty}=\left\langle s_{0}, s_{1}\right\rangle$. The roots of $\Phi$ are the half-lines defined by the 
integers, the positive roots (in $\Phi_{+}$) are those containing $E$. We denote by $a_{0}$ (resp. $a_{1}$ ) the positive root having boundary 0 (resp. 1). Each vertex has type 0 or 1 , with the obvious notation, and the boundary of a root is called its vertex. The type of a root $a$ is the type of its vertex, and is denoted by $\iota(a)$. A positive root $a$ is prenilpotent with $a_{i}$ if and only if $a \supset a_{i}$. Each positive root contains either $a_{0}$ or $a_{1}$, but not both, so the positive roots fall into four disjoint subsets $\mathcal{P}\left(a_{i}, j\right)$ for $i, j \in\{0 ; 1\}$, where $\mathcal{P}\left(a_{i}, j\right)$ is the set of positive roots of type $j$ which contain $a_{i}$. Note that these subsets of positive roots can also be defined as follows:

$$
\begin{array}{ll}
\mathcal{P}\left(a_{0}, 0\right)=\left\{\left(s_{0} s_{1}\right)^{m} a_{0}\right\}_{m \geq 0}, & \mathcal{P}\left(a_{0}, 1\right)=\left\{\left(s_{0} s_{1}\right)^{m} s_{0} a_{1}\right\}_{m \geq 0}, \\
\mathcal{P}\left(a_{1}, 0\right)=\left\{\left(s_{1} s_{0}\right)^{m} s_{1} a_{0}\right\}_{m \geq 0}, & \mathcal{P}\left(a_{1}, 1\right)=\left\{\left(s_{1} s_{0}\right)^{m} a_{1}\right\}_{m \geq 0} .
\end{array}
$$

We shall use $\mathcal{P}\left(a_{i}\right)$ to mean $\mathcal{P}\left(a_{i}, 0\right) \cup \mathcal{P}\left(a_{i}, 1\right)$, the set of positive roots prenilpotent with $a_{i}$.

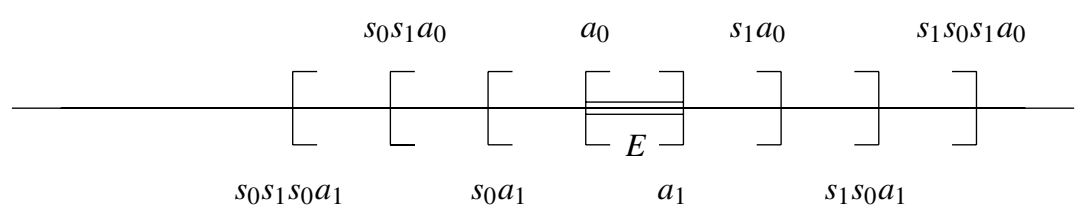

2.B. Borel subgroup. Unipotent subgroups. We pick two fields $\mathbb{K}_{0}$ and $\mathbb{K}_{1}$. To each positive root $a$ of type $i$ is attached a copy of the additive group $\left(\mathbb{K}_{i},+\right)$. We denote it by $U_{a}=\left\{u_{a}(k): k \in \mathbb{K}_{i}\right\}$ ( $u_{a}$ is the chosen isomorphism between the root group and its field).

Definition. (i) Let the abelian group $A_{i}$ be the direct sum of the root groups associated to the roots in $\mathcal{P}\left(a_{i}\right)$.

(ii) Let the group $U_{+}$be the free product of $A_{0}$ and $A_{1}$, that is $U_{+}=A_{0} * A_{1}$.

(iii) Let the group $T$ be the product $\mathbb{K}_{0}^{\times} \times \mathbb{K}_{1}^{\times}$of the multiplicative groups of the chosen fields.

For a (positive) root $a$ of type $i$, we define $\varepsilon_{a} \in\{ \pm 1\}$ to be $(-1)^{m}$, where $m$ is the exponent appearing in 2.A. It is the parity of the number of vertices of type $i$ in the interior of the segment joining the middle of $E$ and the vertex $\partial a$. Using the notation of Example 1 in 1.A, $T$ is the maximal torus

$$
t_{0}\left(\mathbb{K}_{0}^{\times}\right) \times t_{1}\left(\mathbb{K}_{1}^{\times}\right)=\left\{\left(\begin{array}{cc}
\lambda & 0 \\
0 & \lambda^{-1}
\end{array}\right),\left(\begin{array}{cc}
\mu & 0 \\
0 & \mu^{-1}
\end{array}\right): \lambda \in \mathbb{K}_{0}^{\times}, \mu \in \mathbb{K}_{1}^{\times}\right\}
$$

of $\mathrm{SL}_{2}\left(\mathbb{K}_{0}\right) \times \mathrm{SL}_{2}\left(\mathbb{K}_{1}\right)$, and we define the action of $T$ on $U_{+}$by

(2B1) $t_{j}(\lambda) u_{a}(k) t_{j}(\lambda)^{-1}=u_{a}\left(\lambda^{2 \varepsilon_{a} \delta_{j l}(a)} k\right)$ for any $j \in\{0 ; 1\}, \lambda \in \mathbb{K}_{j}^{\times}, a \in \Phi_{+}$ and $k \in \mathbb{K}_{\iota(a)}$, 
and where the Kronecker symbol $\delta_{j \iota(a)}$ means that the element $t_{j}(\lambda)$ induces multiplication of $k$ by $\lambda^{2 \varepsilon_{a}}$ only if the type of the root $a$ is $j$. In particular, $t_{j}(\lambda)$ centralizes each root group of type $\neq j$. The action of a single element $t_{j}(\lambda)$ obviously preserves the relations in $U_{+}$and the actions of any two such elements commute. We therefore obtain an action by $T$ on $U_{+}$.

Definition. (i) The Borel subgroup $B$ is the semi-direct product $T \ltimes U_{+}$.

(ii) We denote by $V^{i}$ the subgroup of $U_{+}$generated by all the positive root groups except $U_{i}$.

(iii) We denote by $U^{i}$ the normal closure of $V^{i}$ in $U_{+}$, i.e. the subgroup generated by $u_{a}(k)$ for $a \in \mathcal{P}\left(a_{i}\right) \backslash\left\{a_{i}\right\}$ and $u_{a_{i}}(r) u_{a}(k) u_{a_{i}}(r)^{-1}$ for $a \in \mathcal{P}\left(a_{1-i}\right)$, with $r \in \mathbb{K}_{i}$.

(iv) The (standard) Levi factor of type $i$ is the direct product $L_{i}=\mathrm{SL}_{2}\left(\mathbb{K}_{i}\right) \times \mathbb{K}_{1-i}^{\times}$.

2.C. Actions of Levi factors on unipotent radicals. In order to define a parabolic subgroup as a semi-direct product $L_{i} \ltimes U^{i}$, we must define an action of $L_{i}$ on the group $U^{i}$. We define actions of $\mathrm{SL}_{2}\left(\mathbb{K}_{i}\right)$ and $\mathbb{K}_{1-i}^{\times}$which are easily seen to commute with one another, so we can deal with each factor separately. The action of the torus $\mathbb{K}_{1-i}^{\times}$ on $U^{i}$ is that obtained as a subgroup of $T$. This makes sense because $T$ obviously stabilizes $U^{i}$. We turn now to the action of the factor $\mathrm{SL}_{2}\left(\mathbb{K}_{i}\right)$. We define the actions of the generators $u_{i}(r)$ and $m_{i}(\lambda)$ given in 1.A.

For the root groups, we set for $k$ in $\mathbb{K}_{0}$ or $\mathbb{K}_{1}$, and $r, s$ in $\mathbb{K}_{i}$ :

(2C1) $u_{i}(r) u_{a}(k) u_{i}(r)^{-1}=u_{a}(k)$ for $a \in \mathcal{P}\left(a_{i}\right) \backslash\left\{a_{i}\right\}$,

(2C2) $u_{i}(s)\left(u_{a_{i}}(r) u_{a}(k) u_{a_{i}}(r)^{-1}\right) u_{i}(s)^{-1}=u_{a_{i}}(r+s) u_{a}(k) u_{a_{i}}(r+s)^{-1}$ for $a \in \mathcal{P}\left(a_{1-i}\right)$.

The elements $m_{i}(\lambda)$ have a conjugation action on the positive root group elements $u_{a}(k)$ :

(2C3) $m_{i}(\lambda) u_{a}(k) m_{i}(\lambda)^{-1}=u_{s_{i} a}\left(\lambda^{-2 \varepsilon_{a} \delta_{i \iota(a)}} k\right)$.

Note that $s_{i} a$ is a non-positive root different from $a_{i}$ since $\Phi_{+} \cap s_{i} \Phi_{-}=\left\{a_{i}\right\}$ [T2, 5.6, Proposition 3]. We must also define a conjugation action of $m_{i}(\lambda)$ on elements $u_{a_{i}}(r) u_{a}(k) u_{a_{i}}(r)^{-1}$ in the free product $U_{a} * U_{a_{i}}$, whenever $a$ is a positive root containing $a_{1-i}$, and $u_{a_{i}}(r) \neq 1$. We set

$$
\text { (2C4) } \begin{aligned}
m_{i}(\lambda) & \left(u_{a_{i}}(r) u_{a}(k) u_{a_{i}}(r)^{-1}\right) m_{i}(\lambda)^{-1} \\
= & u_{a_{i}}\left(\frac{-\lambda^{2}}{r}\right) u_{a}\left(\left(\frac{-\lambda}{r}\right)^{2 \varepsilon_{a} \delta_{i \iota(a)}} k\right) u_{a_{i}}\left(\frac{-\lambda^{2}}{r}\right)^{-1} .
\end{aligned}
$$


Remark. The Kronecker symbol $\delta_{i \iota(a)}$ in the exponents $2 \varepsilon_{a} \delta_{i \iota(a)}$ involves the types of roots $i$ and $\iota(a)$. It simply means that the element $m_{i}(\lambda)$ induces a multiplication of the additive parameter $k$ in $u_{a}(k)$ by a factor $\lambda^{-2 \varepsilon_{a}}$ or $\left(\frac{-\lambda}{r}\right)^{2 \varepsilon_{a}}$ only if the type of the root $a$ is $i$.

2.D. Checking the product relation. In this subsection, we make sure that the individual actions above define an action of $L_{i}$ on $U^{i}$. We must show that given any two elements $g, h$ of $\mathrm{SL}_{2}\left(\mathbb{K}_{i}\right) \times \mathbb{K}_{1-i}$ and a generator $v \in U^{i}$, we always have $g\left(h v h^{-1}\right) g^{-1}=(g h) v(g h)^{-1}$. This equality involves the product $g . h$, and we will have to describe the product of $g$ and $h$ in $\mathrm{SL}_{2}$ in terms of the Bruhat decomposition. When $g$ is in the big cell $\mathrm{SL}_{2}(\underset{\neq 0}{*})$, we write it $g=u_{i}(r) m_{i}(\lambda) u_{i}\left(r^{\prime}\right)$; when it is in the Borel subgroup $\mathrm{SL}_{2}\left(\begin{array}{l}* * \\ 0 *\end{array}\right)$, we write it $g=u_{i}(r) t_{i}(\lambda)$. Similarly, when $h$ is in the big cell, we write it $h=u_{i}(s) m_{i}(\mu) u_{i}\left(s^{\prime}\right)$; when it is in the Borel subgroup, we write it $h=u_{i}(s) t_{i}(\mu)$.

The form of the generator $v$ of $U^{i}$ will also play a role. A generator $v=$ $u_{a_{i}}(t) u_{a}(k) u_{a_{i}}(t)^{-1}$ with $a$ a root containing $a_{1-i}$ (hence not prenilpotent with $a_{i}$ ), will be referred to as a generator of the first type. A generator $v=u_{a}(k)$, with $a$ a root containing $a_{i}$ (hence prenilpotent with $a_{i}$ ), will be referred to as a generator of the second type.

The computation is divided into five cases, according to whether each of the elements $g, h$ or $g h$ belongs to the big cell or its complement the Borel subgroup. Each case is divided into two subcases, according to whether the generator is of the first or of the second type.

We introduce the notation $R=r^{\prime}+s$ and $S=s^{\prime}+t$ for the remainder of Section 2.D.

2.D.1. "big cell - big cell $\in$ big cell", with $g=u_{i}(r) m_{i}(\lambda) u_{i}\left(r^{\prime}\right)$ and $h=$ $u_{i}(s) m_{i}(\mu) u_{i}\left(s^{\prime}\right)$, in which case $R \neq 0$. A calculation in $\mathrm{SL}_{2}$ shows that $g h=$ $u_{i}\left(r-\frac{\lambda^{2}}{R}\right) m_{i}\left(\frac{-\lambda \mu}{R}\right) u_{i}\left(s^{\prime}-\frac{\mu^{2}}{R}\right)$.

We deal with a generator $v$ of the first type. By (2C2) we have $h v h^{-1}=$ $\left[u_{i}(s) m_{i}(\mu)\right] u_{a_{i}}(S) u_{a}(k) u_{a_{i}}(S)^{-1}[\cdots]^{-1}$, and assuming that $S \neq 0$, we have by (2C4) and (2C2) that

$$
\begin{aligned}
& h v h^{-1}=u_{a_{i}}\left(s-\mu^{2} S^{-1}\right) u_{a}\left(\left(-\mu S^{-1}\right)^{2 \varepsilon_{a} \delta_{i \iota(a)}} k\right) u_{a_{i}}(\cdots)^{-1}, \\
& g\left(h v h^{-1}\right) g^{-1}=\left[u_{i}(r) m_{i}(\lambda)\right] u_{a_{i}}\left(R-\mu^{2} S^{-1}\right) \\
& \cdot u_{a}\left(\left(\mu^{2} S^{-1}\right)^{2 \varepsilon_{a} \delta_{i l(a)}} k\right) u_{a_{i}}\left(R-\mu^{2} S^{-1}\right)^{-1}[\cdots]^{-1} .
\end{aligned}
$$


Under the further assumption that $R-\mu^{2} S^{-1} \neq 0,(2 \mathrm{C} 4)$ and (2C2) give

$$
\begin{aligned}
g\left(h v h^{-1}\right) g^{-1}= & u_{a_{i}}\left(r-\frac{\lambda^{2}}{R-\mu^{2} S^{-1}}\right) \\
& \cdot u_{a}\left(\left(\frac{-\lambda}{R-\mu^{2} S^{-1}}\right)^{2 \varepsilon_{a} \delta_{i l(a)}}\left(-\mu S^{-1}\right)^{2 \varepsilon_{a} \delta_{i l(a)}} k\right) \cdot u_{a_{i}}(\cdots)^{-1} \\
= & u_{a_{i}}\left(r-\frac{\lambda^{2} S}{R S-\mu^{2}}\right) \cdot u_{a}\left(\left(\frac{\lambda \mu}{R S-\mu^{2}}\right)^{2 \varepsilon_{a} \delta_{i l(a)}} k\right) \cdot u_{a_{i}}(\cdots)^{-1}
\end{aligned}
$$

Now assuming $\left(R-\mu^{2} S^{-1}\right) \cdot S \neq 0$, we use (2C4) and (2C2) to conjugate $v$ by $g h$ :

$$
\begin{aligned}
(g h) v(g h)^{-1}=[ & \left.u_{i}\left(r-\lambda^{2} R^{-1}\right) m_{i}\left(-\lambda \mu R^{-1}\right)\right] \\
& \cdot u_{a_{i}}\left(S-\mu^{2} R^{-1}\right) u_{a}(k) u_{a_{i}}\left(S-\mu^{2} R^{-1}\right)^{-1}[\cdots]^{-1} \\
= & u_{a_{i}}\left(r-\frac{\lambda^{2}}{R}-\frac{\lambda^{2} \mu^{2}}{R^{2}\left(S-\mu^{2} R^{-1}\right)}\right) \\
& \cdot u_{a}\left(\left(-\frac{-\lambda \mu}{R\left(S-\mu^{2} R^{-1}\right)}\right)^{2 \varepsilon_{a} \delta_{i \iota(a)}} k\right) \cdot u_{a_{i}}(\cdots)^{-1} \\
= & u_{a_{i}}\left(r-\frac{\lambda^{2} S}{R S-\mu^{2}}\right) \cdot u_{a}\left(\left(\frac{\lambda \mu}{R S-\mu^{2}}\right)^{2 \varepsilon_{a} \delta_{i \iota(a)}} k\right) \cdot u_{a_{i}}(\cdots)^{-1},
\end{aligned}
$$

which proves $g\left(h v h^{-1}\right) g^{-1}=(g h) v(g h)^{-1}$ when $S \neq 0$ and $R-\mu^{2} S^{-1} \neq 0$.

Now, suppose $S \neq 0$ but $R-\mu^{2} S^{-1}=0$. Then the first equation above for $g\left(h v h^{-1}\right) g^{-1}$ simplifies, and using $(2 \mathrm{C} 3)$ and $(2 \mathrm{C} 1)$ (which implies that $u_{a_{i}}$ commutes with $u_{s_{i}} a$ ) we obtain

$$
\begin{aligned}
g\left(h v h^{-1}\right) g^{-1} & =u_{a_{i}}(r) u_{s_{i} a}\left(\left(\frac{\mu}{\lambda S}\right)^{2 \varepsilon_{a} \delta_{i l(a)}} k\right) u_{a_{i}}(r)^{-1} \\
& =u_{s_{i} a}\left(\left(\frac{\mu}{\lambda S}\right)^{2 \varepsilon_{a} \delta_{i l(a)}} k\right)
\end{aligned}
$$

and by (2C3) and (2C1) again

$$
\begin{aligned}
(g h) v(g h)^{-1} & =\left[u_{i}\left(r-\lambda^{2} R^{-1}\right) m_{i}\left(-\lambda \mu R^{-1}\right)\right] u_{a}(k)[\cdots]^{-1} \\
& =u_{s_{i} a}\left(\left(\frac{R}{\lambda \mu}\right)^{2 \varepsilon_{a} \delta_{i l(a)}} k\right) .
\end{aligned}
$$

Using $R=\frac{\mu^{2}}{S}$, we obtain the desired equality $g\left(h v h^{-1}\right) g^{-1}=(g h) v(g h)^{-1}$ in this case. 
Finally if $S=0$, by (2C3) and (2C1) we have

$$
\begin{aligned}
h v h^{-1} & =\left[u_{i}(s) m_{i}(\mu)\right] u_{a}(k)[\cdots]^{-1} \\
& =u_{a_{i}}(s) u_{s_{i} a}\left(\frac{k}{\mu^{2 \varepsilon_{a} \delta_{i l(a)}}}\right) u_{a_{i}}(s)^{-1}=u_{s_{i} a}\left(\frac{k}{\mu^{2 \varepsilon_{a} \delta_{i l(a)}}}\right) .
\end{aligned}
$$

By (2C3), $m_{i}(\lambda) u_{s_{i} a}(k) m_{i}(\lambda)^{-1}=u_{a}\left(\lambda^{2 \varepsilon_{a} \delta_{i l}(a)}\right)$. Using this and (2C2),

$$
\begin{aligned}
g\left(h v h^{-1}\right) g^{-1} & =\left[u_{i}(r) m_{i}(\lambda)\right] u_{s_{i} a}\left(\mu^{-2 \varepsilon_{a} \delta_{i \iota(a)}} k\right)[\cdots]^{-1} \\
& =u_{a_{i}}(r) \cdot u_{a}\left(\left(\frac{\lambda}{\mu}\right)^{2 \varepsilon_{a} \delta_{i l(a)}} k\right) \cdot u_{a_{i}}(r)^{-1} .
\end{aligned}
$$

Again with $S=0$, conjugation of $v$ by $g h$ gives by (2C4) and (2C2) that

$$
\begin{aligned}
(g h) v(g h)^{-1}= & {\left[u_{i}\left(r-\lambda^{2} R^{-1}\right) m_{i}\left(-\lambda \mu R^{-1}\right) u_{i}\left(-\mu^{2} R^{-1}\right)\right] u_{a}(k)[\cdots]^{-1} } \\
= & u_{i}\left(r-\frac{\lambda^{2}}{R}\right) u_{a_{i}}\left(\frac{-\lambda^{2} \mu^{2} R^{-2}}{-\mu^{2} R^{-1}}\right) u_{a}\left(\left(\frac{\lambda \mu R^{-1}}{-\mu^{2} R^{-1}}\right)^{2 \varepsilon_{a} \delta_{i \iota(a)}} k\right) \\
& \cdot u_{a_{i}}(\cdots)^{-1} u_{i}(\cdots)^{-1} \\
= & u_{a_{i}}(r) \cdot u_{a}\left(\left(\frac{\lambda}{\mu}\right)^{2 \varepsilon_{a} \delta_{i l(a)}} k\right) \cdot u_{a_{i}}(r)^{-1},
\end{aligned}
$$

hence $g\left(h v h^{-1}\right) g^{-1}=(g h) v(g h)^{-1}$.

The case of a generator $v=u_{a}(k)$ of the second type is simpler, because $a \in$ $\mathcal{P}_{i} \backslash\left\{a_{i}\right\}$, so $u_{a_{i}}$ commutes with $u_{a}$. Using (2C2), (2C3) and (2C4) we obtain

$g\left(h v h^{-1}\right) g^{-1}=(g h) v(g h)^{-1}=u_{a_{i}}\left(r-\frac{\lambda^{2}}{R}\right) \cdot u_{s_{i} a}\left(\left(\frac{R}{\lambda \mu}\right)^{2 \varepsilon_{a} \delta_{i l}(a)}\right) \cdot u_{a_{i}}(\cdots)^{-1}$.

2.D.2. "big cell $\cdot$ big cell $\in$ Borel", with $g$ and $h$ as above. We now have $R=0$ and a calculation in $\mathrm{SL}_{2}$ shows that $g h=u_{i}\left(r+\frac{\lambda^{2}}{\mu^{2}} s^{\prime}\right) t_{i}\left(\frac{-\lambda}{\mu}\right)$. Let $v$ be a generator of the first type. For $g\left(h v h^{-1}\right) g^{-1}$, we compute as in 2.D.1 (with $R=0$ ), the case $S \neq 0$ but $R-\mu^{2} S^{-1}=0$ being excluded. We obtain in any case $g\left(h v h^{-1}\right) g^{-1}=$ $u_{a_{i}}\left(r+\frac{S \lambda^{2}}{\mu^{2}}\right) \cdot u_{a}\left(\left(\frac{\lambda}{\mu}\right)^{2 \varepsilon_{a} \delta_{i l(a)}} k\right) \cdot u_{a_{i}}(\cdots)^{-1}$, which equals $(g h) v(g h)^{-1}$ by $(2 \mathrm{C} 2)$ and (2B1). For a generator of the second type, we have $g\left(h v h^{-1}\right) g^{-1}=(g h) v(g h)^{-1}=$ $u_{a}\left(\left(\frac{\lambda}{\mu}\right)^{2 \varepsilon_{a} \delta_{i l(a)}} k\right)$.

2.D.3. "Borel · big cell $\in$ big cell", with $g=u_{i}(r) t_{i}(\lambda), h=u_{i}(s) m_{i}(\mu) u_{i}\left(s^{\prime}\right)$. A calculation in $\mathrm{SL}_{2}$ shows that $g h=u_{i}\left(r+\lambda^{2} s\right) m_{i}(\lambda \mu) u_{i}\left(s^{\prime}\right)$. 
For a generator of the first type, we have for $S \neq 0$

$$
\begin{aligned}
g\left(h v h^{-1}\right) g^{-1} & =(g h) v(g h)^{-1} \\
& =u_{a_{i}}\left(r+\lambda^{2} s-\frac{\lambda^{2} \mu^{2}}{S}\right) u_{a}\left(\left(\frac{\lambda \mu}{S}\right)^{2 \varepsilon_{a} \delta_{i l(a)}} k\right) u_{a_{i}}(\cdots)^{-1},
\end{aligned}
$$

and for $S=0$

$$
g\left(h v h^{-1}\right) g^{-1}=(g h) v(g h)^{-1}=u_{s_{i} a}\left(\left(\frac{1}{\mu \lambda}\right)^{2 \varepsilon_{a} \delta_{i l}(a)} k\right) .
$$

For a generator of the second type, we have

$$
g\left(h v h^{-1}\right) g^{-1}=(g h) v(g h)^{-1}=u_{a_{i}}\left(r+\lambda^{2} s\right) u_{s_{i} a}\left(\left(\frac{1}{\mu \lambda}\right)^{2 \varepsilon_{a} \delta_{i l}(a)} k\right) u_{a_{i}}(\cdots)^{-1} .
$$

2.D.4. "big cell - Borel $\in$ big cell", with $g=u_{i}(r) m_{i}(\lambda) u_{i}\left(r^{\prime}\right), h=u_{i}(s) t_{i}(\mu)$. A calculation in $\mathrm{SL}_{2}$ shows that $g h=u_{i}(r) m_{i}\left(\frac{\lambda}{\mu}\right) u_{i}\left(\frac{R}{\mu^{2}}\right)$. Write $T=R+\mu^{2} t$, and note that $R \neq 0$ in this case. Let us deal with a generator of the first type. Using (2C) and (2B1) we have $h v h^{-1}=u_{a_{i}}\left(s+\mu^{2} t\right) \cdot u_{a}\left(\mu^{2 \varepsilon_{a} \delta_{i l(a)}} k\right) \cdot u_{a_{i}}(\cdots)^{-1}$. Hence under the assumption that $T \neq 0$, by (2C4) and (2C2) we get $g\left(h v h^{-1}\right) g^{-1}=$ $u_{a_{i}}\left(r-\frac{\lambda^{2}}{T}\right) \cdot u_{a}\left(\left(\frac{\lambda \mu}{T}\right)^{2 \varepsilon_{a} \delta_{i l(a)}} k\right) \cdot u_{a_{i}}(\cdots)^{-1}$, which equals $(g h) v(g h)^{-1}$, since by $(2 \mathrm{C} 4)$ and $(2 \mathrm{C} 2)$ we obtain

$$
\begin{aligned}
(g h) v(g h)^{-1} & =\left[u_{i}(r) m_{i}\left(\lambda \mu^{-1}\right) u_{i}\left(\frac{T}{\mu^{2}}\right)\right] u_{a}(k)[\cdots]^{-1} \\
& =\left[u_{i}(r) u_{i}\left(\frac{-\lambda^{2}}{T}\right)\right] u_{a}\left(\left(\frac{\lambda \mu}{T}\right)^{2 \varepsilon_{a} \delta_{i \iota(a)}} k\right)[\cdots]^{-1}
\end{aligned}
$$

When $T=0$, we simply have

$$
g\left(h v h^{-1}\right) g^{-1}=(g h) v(g h)^{-1}=u_{s_{i} a}\left(\left(\frac{\mu}{\lambda}\right)^{2 \varepsilon_{a} \delta_{i \iota(a)}} k\right) .
$$

For a generator of the second type, we have in any case

$$
g\left(h v h^{-1}\right) g^{-1}=(g h) v(g h)^{-1}=u_{i}(r) \cdot u_{s_{i} a}\left(\left(\frac{\mu}{\lambda}\right)^{2 \varepsilon_{a} \delta_{i \iota(a)}} k\right) \cdot u_{i}(\cdots)^{-1} .
$$

2.D.5. "Borel - Borel $\in$ Borel", with $g=u_{i}(r) t_{i}(\lambda), h=u_{i}(s) t_{i}(\mu)$ and $g h=$ $u_{i}\left(r+\lambda^{2} s\right) t_{i}(\lambda \mu)$. We have $g\left(h v h^{-1}\right) g^{-1}=(g h) v(g h)^{-1}$, equal to $u_{a_{i}}\left(r+\lambda^{2} s+\right.$

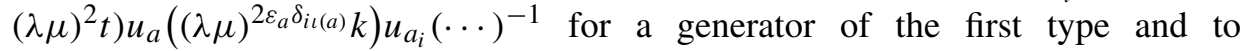
$u_{a}\left((\lambda \mu)^{2 \varepsilon_{a} \delta_{i l(a)}} k\right)$ for a generator of the second type. 
2.E. Twin root datum. Let $i \in\{0 ; 1\}$. By definition of $U^{i}$ as a normal closure, the product map provides a surjective group homomorphism $U_{a_{i}} \ltimes U^{i} \rightarrow U_{+}$. By the very presentation of $U_{+}$, we have another surjective group homomorphism $U_{+} \rightarrow U_{a_{i}} \ltimes U^{i}$ whose composition with the previous one gives the identity automorphism of $U_{+}$. Therefore we have $U_{+} \cong U_{a_{i}} \ltimes U^{i}$.

Definition. (i) We call $P_{i}=L_{i} \ltimes U^{i}$ the (standard) parabolic subgroup of type $i$.

(ii) Associated to the fields $\mathbb{K}_{0}$ and $\mathbb{K}_{1}$, we define the amalgam $\Lambda=P_{0} *_{B} P_{1}$.

Note that (i) makes sense precisely thanks to the computation made in 2.D. By the remark before the definition we have $B \cong\left(T \ltimes U_{a_{i}}\right) \ltimes U^{i}$, and $T \ltimes U_{a_{i}}$ is isomorphic to the upper triangular Borel subgroup $T \ltimes U_{i}$ of the Levi factor $L_{i}=\mathrm{SL}_{2}\left(\mathbb{K}_{i}\right) \times \mathbb{K}_{1-i}^{\times}$. In view of the actions of the subgroups $T=\mathbb{K}_{i}^{\times} \times \mathbb{K}_{1-i}^{\times}$and $U_{i}$ of $L_{i}$ on $U^{i}$, the subgroup $T U_{i} \ltimes U^{i}$ of $P_{i}$ is isomorphic to $B$, and the amalgam in (ii) above makes sense. Moreover by the Bruhat decomposition of $\mathrm{SL}_{2}$, each parabolic subgroup admits the decomposition

$$
P_{i}=B \sqcup U_{i} m_{i} B
$$

for each $i \in\{0 ; 1\}$. We call $N$ the subgroup of $\Lambda$ generated by the elements $m_{i}(\lambda)$ for $i \in\{0 ; 1\}$ and $\lambda$ in $\mathbb{K}_{i}^{\times}$; it is an extension of the Weyl group $D_{\infty}=\left\langle s_{0}, s_{1}\right\rangle$ by $T$, which provides a natural isomorphism $\varphi: W \cong N / T$ under which $s_{i}$ is sent to the class $m_{i}(\lambda) T$. Let now $a=w \cdot a_{i}$ be a root in $\Phi$. The conjugation of a simple root group $U_{a_{i}}$ by $n \in N$ only depends on the class $n T$ (since $T$ normalizes each root group), and in fact $\varphi(w) U_{a_{s}} \varphi(w)^{-1}$ only depends on the root $a$ (by uniqueness of the writing $\left.a=w \cdot a_{i}\right)$. Moreover the action of the elements $m_{i}(\lambda)$ on $U^{i}$ shows that in the case of a positive root $a$, the group $\varphi(w) U_{a_{s}} \varphi(w)^{-1}$ corresponds to the group $U_{a}$ attached to $a$ in the presentation defining $U_{+}(2 . \mathrm{B})$.

Theorem. The group $\Lambda$ satisfies the axioms of a twin root datum for the family of root groups $\left\{U_{a}=u_{a}\left(\mathbb{K}_{l(a)}\right)\right\}_{a \in \Phi}$ above. As a consequence, there exists a semihomogeneous Moufang twin tree of valencies $1+\left|\mathbb{K}_{0}\right|$ and $1+\left|\mathbb{K}_{1}\right|$ for any choice of two fields $\mathbb{K}_{0}$ and $\mathbb{K}_{1}$.

Proof. The group $\Lambda$ is defined as an amalgam, so by [S, I.4, Theorem 7] it acts edgetransitively on the semihomogeneous tree $\Delta$ of valencies $\left[P_{0}: B\right]$ and $\left[P_{1}: B\right]$. By $(\star)$ above, these valencies are $1+\left|\mathbb{K}_{0}\right|$ and $1+\left|\mathbb{K}_{1}\right|$. The stabilizer of an edge (or a vertex of type 0 , resp. of type 1 ) is conjugate to $B$ (or to $P_{0}$, resp. $P_{1}$ ). We have the following identification of $\Lambda$-sets:

$$
\Delta \simeq \Lambda / B \sqcup \Lambda / P_{0} \sqcup \Lambda / P_{1} .
$$

We recover the simplicial structure of the tree $\Delta$ by the reversed inclusion relation on stabilizers. Let us set $t=m_{1} m_{0}$. The set of edges $\left\{t^{n} B\right\}_{n \in \mathbb{Z}} \sqcup\left\{t^{n} m_{1} B\right\}_{n \in \mathbb{Z}}$ defines 
a geodesic in the tree: this is our standard apartment $\mathbb{A}$, containing the standard edge $B$. Let us check the (TRD) axioms. The positive root groups are $t^{-n} U_{0} t^{n}$, $t^{n} m_{1} U_{0} m_{1}^{-1} t^{-n}, t^{n} U_{1} t^{-n}$ or $t^{-n} m_{0} U_{1} m_{0}^{-1} t^{n}$ for $n \geq 0$, and the negative root groups are defined similarly. A pair of roots $\{a ; b\}$ is prenilpotent if $a \supset b$ or $b \supset a$. Conjugating by a suitable power of $t$ allows us to see prenilpotent pairs of roots as pairs of positive roots. Since the group $U_{+}$is defined in such a way that the commutation relations are trivial for all prenilpotent pairs, we get axiom (TRD1). By definition, $\Lambda$ is generated by $P_{0}$ and $P_{1}$, hence by the positive root groups, by $T$ and by the two root groups indexed by the opposite of the simple roots: this is (TRD4). Axiom (TRD2) follows from the relations in $\mathrm{SL}_{2}$ defining the elements $m_{i}(\lambda)$, and from the definition of the root groups. By the definition of $B, T$ normalizes the root groups, which is the second half of axiom (TRD0). By the definition of $\Delta, U_{i}$ is simply transitive on the edges whose closure contains the vertex $P_{i}$ and different from the standard edge: this proves the first assertion of (TRD0) and the second one in (TRD3). The first assertion in (TRD3) follows from what has already been proved [A, §1, Remark 2].

\section{Twinning right-angled buildings}

The previous section provided Moufang twin trees defined by generators and relations. A more general construction is stated in [T4], but for our purposes we need the specific generators and relations of Section 2. In this section we fix a right-angled Coxeter system $(W, S)$, meaning that for all $s_{i}$ and $s_{j}$ in $S$, either $s_{i}$ and $s_{j}$ commute or generate an infinite dihedral group (i.e. $m_{i j}=2$ or $\infty$, so this is a system in which all edges of the diagram are labelled by $\infty$ ). We assume a connected diagram. For the definition of the root system $\Phi$ we refer again to 1.A.

3.A. Root system. For a right-angled Coxeter system $(W, S)$, the Moussong-Davis realization ([D] [Mou]) of the Coxeter complex is a cubical complex. The chamber, which we denote by $C$, is constructed topologically as follows. Let $E_{S}$ be the standard Euclidean vector space of finite dimension $|S|$ with orthonormal basis $\left\{e_{s}\right\}_{s \in S}$. Let $I \subset S$ be a spherical subset of $S$ (in our case a subset such that $W_{I}=\langle s: s \in I\rangle \simeq$ $\left.(\mathbb{Z} / 2 \mathbb{Z})^{|I|}\right)$. Let us consider the subspace $E_{I}$ generated by the vectors $e_{s}$ for $s \in I$. We denote by $C_{I}$ the cube generated by the $2^{|I|}$ vectors of $E_{I}$ with coordinates equal to 0 or 1 . Then the (closed) Moussong-Davis chamber $C$ is the union in $E_{S}$ of all the cubes $C_{I}$ when $I$ runs over the spherical subsets of $S$. Its panel $\Pi_{s}$ of type $\{s\}$ is the intersection of $C$ with the hyperplane of vectors of coordinate 1 along $e_{s}$.

Vertices in $C$ correspond to maximal spherical subsets of $S$ and the inclusion of a canonical generator $s$ in a maximal spherical subset $I$ (with at least two elements) corresponds to the inclusion of a vertex $v_{I}$ in the panel $\Pi_{s}$. The sub- 
group $\langle t: t \in I \backslash\{s\}\rangle \simeq(\mathbb{Z} / 2 \mathbb{Z})^{|I|-1}$ acts simple transitively on the panels of type $s$ around $v_{I}$ in the Coxeter complex and preserves $\partial a_{s}$. This implies that all panels in the boundary wall $\partial a_{s}$ have the same type, and that the Coxeter subgroup $W^{\{s\}}=\langle j: j \in S \backslash\{s\}$ and $[j, s]=1\rangle$ acts transitively on the panels in $\partial a_{s}$. The latter fact also implies that $\operatorname{Stab}_{W}\left(\partial a_{s}\right)=W^{\{s\}} \times\langle s\rangle$ and $\operatorname{Stab}_{W}\left(a_{s}\right)=W^{\{s\}}$ (since if $w \in \operatorname{Stab}_{W}\left(\partial a_{s}\right)$ then there is $w^{\prime} \in W^{\{s\}}$ such that $\left.\left(w^{\prime}\right)^{-1} w \cdot \Pi_{s}=\Pi_{s}\right)$.

We have seen that given a root $a$ the panels on its boundary wall all have the same type. We shall refer to this as the type of the root $a$, and denote it by $\iota(a) \in S$. Finally for a root $a$, we define $\varepsilon_{a} \in\{ \pm 1\}$ to be $(-1)^{m}$, where $m$ is the number of walls of type $\iota(a)$ meeting the interior of any geodesic segment from a point $x \in C \backslash \bigcup_{s \in S} \Pi_{s}$ to the wall $\partial a$.

Example. Let us consider a regular right-angled $r$-gon $R$ of the hyperbolic plane $\mathbb{H}^{2}$.

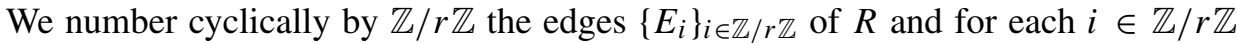
we let $s_{i}$ denote the reflection in the geodesic supporting $E_{i}$. Then the subgroup $W$ of $\mathrm{PSL}_{2}(\mathbb{R})$ generated by the reflections $s_{i}$ is a Coxeter group, and $\left(W,\left\{s_{i}\right\}_{i \in \mathbb{Z} / r \mathbb{Z})}\right.$ is a right-angled Coxeter system. In the above construction, the chamber $C$ is the union in $\mathbb{R}^{r}$ of $r$ squares $C_{\{i ; i+1\}}$, and each panel $\Pi_{i}$ is the union of two edges, one in $C_{\{i-1 ; i\}}$ and one in $C_{\{i ; i+1\}}$. To recover the original tiling of $\mathbb{H}^{2}$ by $R$, we can so to speak "hyperbolize" each Euclidean square $C_{\{i ; i+1\}}$ into a hyperbolic square, by replacing the right angle of $C_{\{i ; i+1\}}$ at the origin of $\mathbb{R}^{r}$ by an angle $\frac{2 \pi}{r}$.

3.B. Borel subgroup. Unipotent radicals. For each $s \in S$, we pick a field $\mathbb{K}_{s}$. To each positive root $a$ of type $s$ is attached a copy of the additive group $\left(\mathbb{K}_{s},+\right)$. We denote it by $U_{a}=\left\{u_{a}(k): k \in \mathbb{K}_{s}\right\}$, where $u_{a}$ is a chosen isomorphism from $\left(\mathbb{K}_{s},+\right)$ to $U_{a}$.

Definition. (i) We define the standard torus to be the direct product $T=\prod_{s \in S} \mathbb{K}_{s}^{\times}$ of the multiplicative groups of the chosen fields.

(ii) We define the group $U_{+}$by generators and relations: it is the quotient of the free product $*_{a \in \Phi_{+}} U_{a}$ by the relations $\left[U_{a}, U_{b}\right]=\{1\}$ whenever $\{a ; b\}$ is prenilpotent.

We view $T$ as the maximal torus $\prod_{s \in S} t_{s}\left(\mathbb{K}_{s}^{\times}\right)$of $\prod_{s \in S} \mathrm{SL}_{2}\left(\mathbb{K}_{s}\right)$ (the notation $t_{s}$ refers to the same parametrization of the diagonal matrices of $\mathrm{SL}_{2}$ as in Example 1 of 1.A). As in (2B1), we make $T$ act on $U_{+}$by

$$
t_{S}(\lambda) u_{a}(k) t_{S}(\lambda)^{-1}=u_{a}\left(\lambda^{2 \varepsilon_{a} \delta_{s \iota}(a)} k\right)
$$

for any $s \in S$, any $a \in \Phi_{+}$and any $\lambda \in \mathbb{K}_{s}^{\times}$and $k \in \mathbb{K}_{\iota(a)}$. In particular, $t_{s}(\lambda)$ centralizes any root group of type $\neq s$.

Definition. We define the (standard) Borel subgroup $B$ to be the semi-direct product $T \ltimes U_{+}$. 
We can now introduce unipotent radicals. Let $I$ be a spherical subset of $S$, and let $R(I)$ denote the corresponding set of simple roots $\left\{a_{i}\right\}_{i \in I}$. Thus for any two $i, j \in I$, we have $\left[U_{a_{i}}, U_{a_{j}}\right]=\{1\}$.

Definition. We denote by $V^{I}$ the subgroup of $U_{+}$generated by all the positive root groups except the groups $U_{a_{i}}$ for $i \in I$, and we denote by $U^{I}$ the normal closure of $V^{I}$ in $U_{+}$.

For any positive root $a \notin R(I)$, we set $I(a)=\left\{j \in I: a_{j}\right.$ is not prenilpotent with $a$ \}. Then for any choice of parameters $k_{a} \in \mathbb{K}_{\iota(a)}$ and $k_{j} \in \mathbb{K}_{j}, j \in I(a)$, the element

$$
\left(\prod_{j \in I(a)} u_{a_{j}}\left(k_{j}\right)\right) \cdot u_{a}\left(k_{a}\right) \cdot\left(\prod_{j \in I(a)} u_{a_{j}}\left(k_{j}\right)\right)^{-1}
$$

lies in $U^{I}$, and the family of all such elements is a generating system for $U^{I}$.

3.C. Actions of Levi factors on unipotent radicals. We first define the Levi factors.

Definition. Let $I$ be a spherical subset of $S$. We define the (standard) Levi factor of type $I$ to be the group $L_{I}=\prod_{s \in I} \mathrm{SL}_{2}\left(\mathbb{K}_{s}\right) \times \prod_{s \notin I} t_{s}\left(\mathbb{K}_{s}^{\times}\right)$.

Let us define now the action of individual elements in the group $L_{I}$ on $U^{I}$. First, the $T$-action was defined in 3.B (its action on $U_{+}$restricts to $U^{I}$ ). Let $v=$ $\left(\prod_{j \in I(a)} u_{a_{j}}\left(k_{j}\right)\right) \cdot u_{a}\left(k_{a}\right) \cdot\left(\prod_{j \in I(a)} u_{a_{j}}\left(k_{j}\right)\right)^{-1}$ be an element of $U^{I}$ in the form given in 3.B. Let us pick $s \in I$ and $k \in \mathbb{K}_{s}$. If $s \notin I(a)$ we assume that $u_{s}(k)$ centralizes $v$. Otherwise, we define $u_{s}(k) v u_{s}(k)^{-1}$ to be

$$
\left(\prod_{j \in I(a) \backslash\{s\}} u_{a_{j}}\left(k_{j}\right)\right) u_{a_{s}}\left(k_{s}+k\right) \cdot u_{a}\left(k_{a}\right) \cdot u_{a_{s}}\left(k_{s}+k\right)^{-1}\left(\prod_{j \in I(a) \backslash\{s\}} u_{a_{j}}\left(k_{j}\right)\right)^{-1} .
$$

Let us now pick $s \in I$ and $\lambda \in \mathbb{K}_{s}^{\times}$. If $s \notin I(a)$ or if $s \in I(a)$ but $k_{s}=0$, we set

$$
m_{s}(\lambda) v m_{s}(\lambda)^{-1}=\left(\prod_{j \in I(a)} u_{a_{j}}\left(k_{j}\right)\right) \cdot u_{s(a)}\left(\lambda^{-2 \varepsilon_{a} \delta_{s \iota}(a)} k_{a}\right) \cdot\left(\prod_{j \in I(a)} u_{a_{j}}\left(k_{j}\right)\right)^{-1} .
$$

Otherwise, i.e. if $s \in I(a)$ and $k_{s} \neq 0$, we define $m_{s}(\lambda) v m_{s}(\lambda)^{-1}$ to be

$$
\begin{aligned}
& \left(\prod_{j \in I(a) \backslash\{s\}} u_{a_{j}}\left(k_{j}\right)\right) u_{a_{s}}\left(\frac{-\lambda^{2}}{k_{s}}\right) \\
& \quad \cdot u_{a}\left(\left(\frac{-\lambda}{k_{s}}\right)^{2 \varepsilon_{a} \delta_{s l(a)}} k_{a}\right) \cdot u_{a_{s}}\left(\frac{-\lambda^{2}}{k_{s}}\right)^{-1}\left(\prod_{j \in I(a) \backslash\{s\}} u_{a_{j}}\left(k_{j}\right)\right)^{-1} .
\end{aligned}
$$

In other words, up to the conjugating element $\left(\prod_{j \in I(a) \backslash\{s\}} u_{a_{j}}\left(k_{j}\right)\right)$, the formulas are copied from the tree case (2.C). 
3.D. Checking the product relation. We now check that these individual actions provide an action by $L_{I}$; this is done by checking that $g\left(h v h^{-1}\right) g^{-1}=(g h) v(g h)^{-1}$ for any generator $v \in U^{I}$ and any $g, h \in L_{I}$. The Levi factor of type $I$ is a direct product and the actions of different factors in 3.C are easily seen to commute with one another, so it is enough to check each factor separately. We are reduced to showing that for a single factor $\mathrm{SL}_{2}\left(\mathbb{K}_{S}\right)$, the actions of $u_{s}(k), t_{s}(\lambda)$ and $m_{s}(\lambda)$ as above provide an $\mathrm{SL}_{2}\left(\mathbb{K}_{s}\right)$-action. We noted in 3.C that the formulas defining the individual actions are the same as those of the tree case up to conjugation by elements of the form $\left(\prod_{j \in I(a) \backslash\{s\}} u_{a_{j}}\left(k_{j}\right)\right)$, which are all centralized by $\mathrm{SL}_{2}\left(\mathbb{K}_{s}\right)$. Therefore the latter verification follows from the computation made in 2.D. This finally shows that the Levi factor $L_{I}$ acts on the unipotent radical $U^{I}$.

Definition. Let $I$ be a spherical subset of $S$. We define the (standard) parabolic subgroup of type $I$ to be $P_{I}=L_{I} \ltimes U^{I}$.

The definition of $U^{I}$ in 3.B implies that $U_{+}=U_{I} \cdot U^{I}$, where $U^{I} \triangleleft U_{+}$and $U_{I}=\prod_{s \in I} U_{a_{s}}$. This provides a surjective group homomorphism $U_{I} \ltimes U^{I} \rightarrow$ $U_{+}$. The presentation defining $U_{+}$implies the existence of another surjective group homomorphism $U_{+} \rightarrow U_{I} \ltimes U^{I}$, and the composition of these two homomorphisms is the identity map of $U_{+}$. Therefore we have $U_{+} \cong U_{I} \ltimes U^{I}$ for any spherical subset $I$ of $S$. This implies that for an inclusion $J \subset I$ of spherical subsets, we have $U^{J} \cong U_{I \backslash J} \ltimes U^{I}$ where $U_{I \backslash J}=\prod_{s \in I \backslash J} U_{a_{s}}$.

3.E. Twin root datum. We consider the group

$$
P_{I}^{J}=\prod_{s \in J} \mathrm{SL}_{2}\left(\mathbb{K}_{s}\right) \times \prod_{s \in I \backslash J}\left(t_{s}\left(\mathbb{K}_{s}^{\times}\right) \ltimes U_{s}\right) \times \prod_{s \notin I} t_{s}\left(\mathbb{K}_{s}^{\times}\right),
$$

which is a standard parabolic subgroup of the Levi factor $L_{I}$, containing the Levi factor $L_{J}$. In the middle factor, the group $t_{s}\left(\mathbb{K}_{s}^{\times}\right) \ltimes U_{s}$ is the upper triangular Borel subgroup of $\mathrm{SL}_{2}\left(\mathbb{K}_{S}\right)$. In the language of parabolic subgroups of reductive groups, the group $\prod_{s \in I \backslash J} U_{s}$ is the unipotent radical of the parabolic subgroup $P_{I}^{J}$ of $L_{I}$, and we can also write

$$
P_{I}^{J}=L_{J} \ltimes \prod_{s \in I \backslash J} U_{s} .
$$

We have

$$
P_{I}=L_{I} \ltimes U^{I} \supset P_{I}^{J} \ltimes U^{I}=\left(L_{J} \ltimes \prod_{s \in I \backslash J} U_{s}\right) \ltimes U^{I} \cong L_{J} \ltimes\left(\prod_{s \in I \backslash J} U_{s} \ltimes U^{I}\right) .
$$

The group $\prod_{s \in I \backslash J} U_{s}$ lies in the Levi factor $L_{I}$ whereas the group $U_{I \backslash J}=$ $\prod_{s \in I \backslash J} U_{a_{s}}$ of the previous subsection lies in $U^{J}$. But these two groups are naturally isomorphic and under the isomorphism, the $L_{I}$-action on $U^{I}$ (defined in 3.C) 
restricted to $\prod_{s \in I \backslash J} U_{s}$ is the $U_{I \backslash J}$-action on $U^{I}$ by conjugation given by the presentation of $U_{+}$. By $U^{J} \cong U_{I \backslash J} \ltimes U^{I}$ (end of 3.D), this shows that for any inclusion $J \subset I$ as above we have an injective group homomorphism $P_{J} \hookrightarrow P_{I}$. Finally, varying such inclusions we can see the finite set of standard spherical parabolic subgroups as an inductive system of groups with injective transition maps.

Definition. (i) We denote by $\Lambda$ the limit of the inductive system indexed by the inclusions of spherical subsets $I$, where the groups are the corresponding parabolic subgroups $P_{I}$.

(ii) We denote by $N$ its subgroup generated by the elements $m_{s}(\lambda)$ when $s$ varies in $S$ and $\lambda$ in $\mathbb{K}_{S}^{\times}$.

Remarks. 1) Since any transition map of the inductive system is injective, any parabolic subgroup $P_{I}$ is naturally a subgroup of $\Lambda$.

2) In the terminology of [HP, Section 5], $\Lambda$ is the fundamental group of the complex of groups indexed by the cone over the first barycentric subdivision of the finite nerve of the Coxeter system $(W, S)$. This complex of groups is described precisely in the remark following [loc. cit., Proposition 5.1], and to recover our situation it is enough to replace the group $G_{J}$ therein by the parabolic subgroup $P_{J}$ (with the convention that $B=P_{\varnothing}$ ).

In view of the relation $m_{s}(\lambda) m_{s}(-1)=t_{s}(\lambda)$, we see that $N$ contains $T$, and in fact $T \triangleleft N$. For each spherical subset $I$ we denote by $N_{I}$ the subgroup of the Levi factor $L_{I}$ generated by $T$ and the elements $m_{s}(1), s \in I$. Then we obtain another inductive system indexed by the spherical subsets, whose limit is $N$. The Coxeter group $W$ itself is also the limit of the inductive system obtained from the previous one by factoring out by the intersections with $T$, and there is a natural isomorphism $\varphi: W \cong N / T$ under which $s$ is sent to the class $m_{s}(\lambda) T$.

Now let $a=w \cdot a_{s}=w^{\prime} \cdot a_{t}$ be a root of $\Phi$. Since we are dealing with right-angled Coxeter groups (3.A) we have $s=t$ and $w^{-1} w^{\prime} \in W^{\{s\}}=\langle j: j \in S \backslash\{s\}$ and $[j, s]=1\rangle$. As usual we can identify $w$ with a coset of $T$ in $N$, and since $T$ normalizes the root groups $U_{a_{s}}$, the conjugate $w U_{a_{s}} w^{-1}$ is well-defined. Therefore we can write

$$
w^{\prime} U_{a_{t}} w^{-1}=w\left(w^{-1} w^{\prime}\right) U_{a_{s}}\left(w^{-1} w^{\prime}\right)^{-1} w^{-1}=w U_{a_{s}} w^{-1},
$$

which shows that $w U_{a_{s}} w^{-1}$ only depends on the root $a$. Moreover the action of the elements $m_{s}(\lambda)$ on $U^{\{s\}}$ shows that in the case of a positive root $a$, the group $w U_{a_{s}} w^{-1}$ corresponds to the group $U_{a}$ attached to $a$ in the presentation defining $U_{+}$(3.B).

Our main constructive result about twinnings of right-angled buildings can now be stated.

Theorem. (i) The triple $\left(\Lambda,\left\{U_{a}\right\}_{a \in \Phi}, T\right)$ is a twin root datum in which for any prenilpotent pair of roots the corresponding root groups commute. 
(ii) Every right-angled building with a parameter system of cardinalities of projective lines belongs to a Moufang twinning.

Proof. (i) We have to prove axioms (TRD0) to (TRD4) of 1.A, together with the improvement of (TRD1) on the trivial commutation of root groups. (TRD0) and the second part of (TRD2) are clear by the discussion preceding the statement of the theorem, where the subgroup $N$ and the root groups are defined. The first part of (TRD2) follows from a simple computation in $\mathrm{SL}_{2}: m_{s}(\lambda)=u_{-a_{s}}\left(\lambda^{-1}\right) u_{a_{s}}(\lambda) u_{-a_{s}}\left(\lambda^{-1}\right)$ (see Example 1 in 1.A) and [T5, comment following 3.3] for the uniqueness assertion). Let now $\{a ; b\}$ be a prenilpotent pair of roots. There is $w \in W$ such that $w .\{a ; b\} \subset \Phi_{+}$, so that the conjugates $\varphi(w) U_{a} \varphi(w)^{-1}$ and $\varphi(w) U_{b} \varphi(w)^{-1}$ lie in $U_{+}$, in which the commutation relation is trivial by definition. This proves the improvement of (TRD1). The parabolic subgroup $P_{\{s\}}$ injects in $\Lambda$ and we have $P_{\{s\}}=U_{a_{s}} m_{s} B \sqcup B$ with $U_{-a_{s}} \backslash\{1\} \subset U_{a_{s}} m_{s} B$. This proves the second assertion of (TRD3) and the first assertion follows from all the other axioms [A, §1, Remark 2]. It remains to prove (TRD4). This is clear because $\Lambda$ is generated by the parabolic subgroups $P_{I}$ and any such group is generated by the root groups it contains.

(ii) The twin root datum structure implies that $\Lambda$ naturally acts on a twin building $\Delta_{ \pm}$with Weyl group $W \cong N / T$ and that $\Delta_{ \pm}$enjoys the Moufang property [T5]. Moreover any residue of type $\{s\}, s \in S$, is isomorphic to the building $\mathbb{P}^{1}\left(\mathbb{K}_{s}\right)$. But it follows from [HP, Proposition 5.1] that, up to isomorphism, there is a unique rightangled building of given Coxeter type $(W, S)$ and parameter system. This uniqueness implies (ii) when the parameter system consists of cardinalities of projective lines.

Remark. In 4.B, we will need a slight modification of the construction. We will need to replace the group $\mathrm{SL}_{2}$ by the group $\mathrm{GL}_{2}$, in order to obtain local actions on residues of panels via $\mathrm{PGL}_{2}$ instead of $\mathrm{PSL}_{2}$. This can be done as follows. In the definition of $T$ (3.B), each factor $t_{s}\left(\mathbb{K}_{s}^{\times}\right)=\left\{\left(\begin{array}{cc}\lambda & 0 \\ 0 & \lambda^{-1}\end{array}\right): \lambda \in \mathbb{K}_{s}^{\times}\right\}$can be replaced by the group $\left\{d_{s}(\lambda, \mu)=\left(\begin{array}{ll}\lambda & 0 \\ 0 & \mu\end{array}\right): \lambda, \mu \in \mathbb{K}_{s}^{\times}\right\}$, and in the definition of the Levi subgroups $L_{I}$, each factor $\mathrm{SL}_{2}\left(\mathbb{K}_{S}\right)$ can be replaced by a factor $\mathrm{GL}_{2}\left(\mathbb{K}_{S}\right)$ (3.C). In the actions given in 3.B and 3.C, the only change concerns the diagonal matrices of $\mathrm{GL}_{2}\left(\mathbb{K}_{s}\right)$ acting on root groups, for which we shall set the formula

$$
d_{s}(\lambda, \mu) u_{a}(k) d_{s}(\lambda, \mu)^{-1}=u_{a}\left(\left(\frac{\lambda}{\mu}\right)^{\varepsilon_{a} \delta_{s l}(a)} k\right)
$$

for any $s \in S$, any $a \in \Phi_{+}$and any $\lambda, \mu \in \mathbb{K}_{s}^{\times}$and $k \in \mathbb{K}_{\iota(a)}$. This generalizes the formula $t_{s}(\lambda) u_{a}(k) t_{s}(\lambda)^{-1}=u_{a}\left(\lambda^{2 \varepsilon_{a} \delta_{s l}(a)} k\right)$ of 3.B since $t_{s}(\lambda)=d_{s}\left(\lambda, \lambda^{-1}\right)$. Then the group $\Lambda$ generated by $T$ and the root groups has a twin root datum structure $\left(\Lambda,\left\{U_{a}\right\}_{a \in \Phi}, T\right)$ with Weyl group the right-angled Coxeter group $W$. 


\section{Non-linearities}

A Kac-Moody group defined over $\mathbb{F}_{q}$ has no reason to be linear in characteristic $p$ (where $q$ is a power of $p$ ) merely because it is defined by a presentation involving subgroups that are linear over $\mathbb{F}_{q}$. Moreover an infinite Kac-Moody group over $\mathbb{F}_{q}$ cannot be linear over any field of characteristic different from $p$ [Ré3]. In Sections 2 and 3 we constructed groups of root datum type with more than one ground field and we now show that if these fields have different characteristics, then the group cannot be linear.

4.A. Non-linearity of Borel subgroups. Our result actually shows that even much smaller subgroups of the alluded to above groups have strong non-linearity properties.

Theorem. Let $(W, S)$ be a right-angled Coxeter system with Coxeter diagram $D$. Let $\left\{\mathbb{K}_{s}\right\}_{s \in S}$ be a choice of fields with two fields $\mathbb{K}_{s}$ and $\mathbb{K}_{t}$ of different characteristics for $s \neq t$ in the same connected component of $D$. Let $\Lambda$ be the corresponding group defined in 3.E and let $\Gamma$ be the fixator of some chamber in the associated twinned buildings. Then, any group homomorphism $\rho: \Gamma \rightarrow \prod_{\alpha \in A} \mathbb{G}_{\alpha}\left(\mathbb{F}_{\alpha}\right)$ has infinite kernel, whenever the index set $A$ is finite and $\mathbb{G}_{\alpha}$ is a linear algebraic group defined over the field $\mathbb{F}_{\alpha}$ for each $\alpha \in A$.

Proof. Any two chamber-fixators are isomorphic, so we deal with the standard positive chamber $C$ (3.A). Its fixator is $B=T \ltimes U_{+}$(3.B), and we shall use the notation $B$ rather than $\Gamma$. Let $\mathcal{C}$ be the connected component of the vertices $s$ and $t$ in the Coxeter diagram of $(W, S)$. We let $\mathcal{C}_{s}$ denote the subset of vertices in $\mathcal{C}$ to which are attached fields having the same characteristic as $\mathbb{K}_{s}$. The complement $\mathcal{C} \backslash \mathcal{C}_{s}$ is non-empty since it contains $t$. Taking an edge connecting $\mathcal{C}_{s}$ to $\mathcal{C} \backslash \mathcal{C}_{s}$ we obtain an edge with one vertex, say $i$, with corresponding field $\mathbb{K}_{i}$ of characteristic $p_{i}$ and the other vertex, say $j$, with corresponding field $\mathbb{K}_{j}$ of characteristic $p_{j} \neq p_{i}$.

Notice that $s_{i}$ and $s_{j}$ generate an infinite dihedral group; set $t=s_{j} s_{i}$. To simplify notation the root $a_{s_{i}}$ will be denoted $a_{i}$. For any integer $m \geq 0$, the root $a_{i}(m)=$ $t^{m} .\left(s_{j} \cdot a_{i}\right)$ is a positive root of $\Phi$. Similarly, for any integer $m \geq 0$ we set $a_{j}(m)=$ $t^{-m} .\left(s_{i} . a_{j}\right)$ and we have $a_{j}(m) \in \Phi_{+}$. For any $m^{\prime} \geq m$ we have $a_{i}\left(m^{\prime}\right) \supset a_{i}(m)$, implying that the pair of roots $\left\{a_{i}\left(m^{\prime}\right) ; a_{i}(m)\right\}$ is prenilpotent. In view of the defining relations of $\Lambda$, the subgroup of $B$ generated by the root groups $U_{a_{i}(m)}, m \geq 0$, is the direct sum of countably many copies of $\mathbb{K}_{i}$; so it is an infinite group of exponent $p_{i}$, which we denote by $V_{i}$. Similarly, the group $V_{j}=\left\langle U_{a_{j}(m)}: m \geq 0\right\rangle$ is an infinite subgroup of exponent $p_{j}$ in $B$.

We set $A_{i}=\left\{\alpha \in A: \operatorname{char}\left(\mathbb{F}_{\alpha}\right)=p_{i}\right\}$ and $A_{j}=\left\{\alpha \in A: \operatorname{char}\left(\mathbb{F}_{\alpha}\right)=p_{j}\right\}$. For each $\alpha \in A$, let $\mathrm{pr}_{\alpha}: \prod_{\alpha \in A} \mathbb{G}_{\alpha}\left(\mathbb{F}_{\alpha}\right) \rightarrow \mathbb{G}_{\alpha}\left(\mathbb{F}_{\alpha}\right)$ be the natural projection. By [Mar, VIII.3.7] for each $\alpha \notin A_{i}$ the group $\left(\mathrm{pr}_{\alpha} \circ \rho\right)\left(V_{i}\right)$ is finite, so there is a finite index normal subgroup $N_{i} \triangleleft V_{i}$ such that $\prod_{\alpha \notin A_{i}}\left(\mathrm{pr}_{\alpha} \circ \rho\right)\left(N_{i}\right)$ is trivial, and similarly 
for $j$ replacing $i$. The subgroup generated by $U_{a_{i}}, U_{a_{j}}, U_{-a_{i}}=m_{i} U_{a_{i}} m_{i}^{-1}$ and $U_{-a_{j}}=m_{j} U_{a_{j}} m_{j}^{-1}$ satisfies the axioms of a twin root datum with infinite dihedral Weyl group, and by applying [KP, Proposition 3.5 (c)] to the corresponding refined Tits system (1.A), we have a free product decomposition of its subgroup generated by the positive root groups of type $i$ and $j$. This shows that $V_{i} * V_{j}$ injects in $B$, and if we pick $v \in N_{i} \backslash\{1\}$ and $v^{\prime} \in N_{j} \backslash\{1\}$, then $v v^{\prime}$ has infinite order. But since $v \in N_{i}$ and $v^{\prime} \in N_{j}$ the images $\rho(v)$ and $\rho\left(v^{\prime}\right)$ commute with one another and both have finite order. We have found in $B$ a subgroup isomorphic to $\mathbb{Z}$ with finite image under $\rho$.

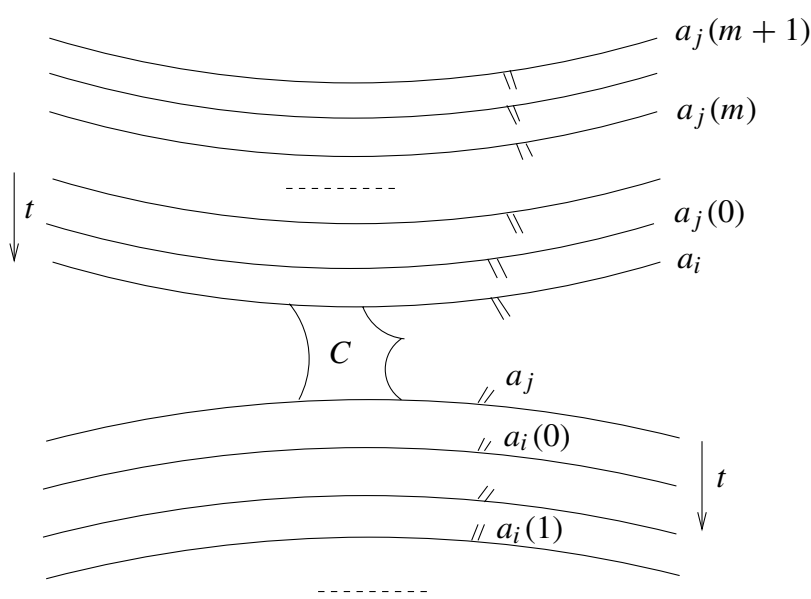

If we assume that each field $\mathbb{K}_{s}$ in the theorem is a finite field $\mathbb{F}_{q_{s}}$, then we are in the situation of 1.B and 1.C: we have a locally finite building $\Delta_{+}=\Delta$ and a locally compact group $G$ (1.B), which we see as a completion of $\Lambda$.

Recall moreover that a discrete subgroup $D$ in a topological group $H$ is called a lattice if the homogeneous space $H / D$ carries a finite $H$-invariant measure [Mar, I.0.40]. A lattice $D<H$ is called uniform if the quotient space $H / D$ is compact. The theory of lattices in Lie groups shows that it is extremely useful to see an abstract discrete group $D$ as a lattice in a topological group $H$ (with a much richer structure). The connection with our specific situation is the following. Let $W(t)$ denote the growth series of the Weyl group $W$ with respect to the generating system $S$, meaning the power series $\sum_{n \geq 0} c_{n} t^{n}$ where $c_{n}$ is the number of words of length $n$. If $W\left(\frac{1}{q}\right)$ converges for $q=\min _{s \in S} q_{s}$, then this implies that any spherical negative parabolic subgroup $\Gamma$ of $\Lambda$ is a (non-uniform) lattice of $G$ [Ré2]. Combining this with the previous theorem provides the next result.

Corollary. In the same situation as in the theorem, we assume further that each field $\mathbb{K}_{s}$ is a finite field $\mathbb{F}_{q_{s}}$, that $\Gamma$ is the fixator of some negative facet and that 
$q=\min _{s \in S} q_{s}$ is large enough so that $\Gamma$ is a lattice in $G$. Then, any group homomorphism $\rho: \Gamma \rightarrow \prod_{\alpha \in A} \mathbb{G}_{\alpha}\left(\mathbb{F}_{\alpha}\right)$ as in the theorem has infinite kernel.

We therefore obtain a wide family of group inclusions $\Gamma<G$ generalizing lattices in Lie groups, the difference being that no group here is linear. Non-linearity follows from the use of ground fields of different characteristics (which is not available in the case of a Kac-Moody group over a finite field), and uses our construction of exotic twin root data in 3.E. Linearity problems for Kac-Moody groups over finite fields are considered in [Ré5], but the results in the latter work concern the full Kac-Moody groups $\Lambda$ rather than their parabolic subgroups $\Gamma$.

4.B. Two kinds of lattices. In this last subsection we show that, in some cases, the topological group $G$ of the corollary not only contains non-linear lattices but also lattices which are linear in characteristic 0 . For this we assume that, given $(W, S)$ and $\left\{\mathbb{F}_{q_{s}}\right\}_{s \in S}$ as in the corollary, the group $\Lambda$ is defined by means of the modification described in the concluding remark of 3.E. This is to have the group $\Lambda$ acting locally on residues of panels of type $s$ via $\mathrm{PGL}_{2}\left(\mathbb{F}_{q_{s}}\right)$ rather than via $\mathrm{PSL}_{2}\left(\mathbb{F}_{q_{s}}\right)$.

Lemma. (i) The group $\Lambda$ acts on a right-angled twin building $\Delta_{ \pm}$, where $\Delta_{ \pm}$is the unique building of type $(W, S)$ and parameter system $\left\{1+q_{s}\right\}_{s \in S}$.

(ii) The kernel of the action on each building $\Delta_{ \pm}$is the finite center $Z(\Lambda)$.

Proof. (i) follows from 3.E and uniqueness of the buildings under consideration [HP, Proposition 5.1]. (ii) follows from Tits system arguments as in [Ré1, 8.4.3]. In particular, $Z(\Lambda)$ is the subgroup of $T$ made of the elements centralizing each root group. Note that it is the product $\prod_{s \in S} Z\left(\mathrm{GL}_{2}\left(\mathbb{F}_{q_{s}}\right)\right) \cong \prod_{s \in S} \mathbb{F}_{q_{s}}^{\times}$, so it intersects trivially the group $U_{+}$, and hence any unipotent radical.

By [GP] and the remark before the proof of [HP, Proposition 5.1] about complexes of groups, $\Delta_{ \pm}$is the universal cover of a simple complex of groups indexed by a cone over the barycentric subdivision of the finite nerve of $(W, S)$. In this theory, the building $\Delta_{ \pm}$is constructed together with a uniform lattice which is the limit of an inductive system indexed by the same directed set as in 3.E (the set of spherical subsets), but in which the groups can be chosen to be much simpler (e.g. finite) than the parabolic subgroups. The simplest choice is when the parabolic subgroup $P_{I}$ is replaced by the product $\prod_{s \in I} \mathbb{Z} /\left(q_{s}+1\right) \mathbb{Z}$, with the obvious inclusions as transition maps. We call $\Gamma_{(W, S), 1+q}$ the uniform lattice defined by this choice of groups.

Proposition. The subgroup $\Lambda / Z(\Lambda)$ of $\operatorname{Aut}\left(\Delta_{ \pm}\right)$contains the uniform lattice $\Gamma_{(W, S), 1+q}$.

Proof. The fixator in $\Lambda / Z(\Lambda)$ of the standard facet of spherical type $I$ admits a semidirect product decomposition $L_{I} / Z(\Lambda) \ltimes U^{I}$, with $L_{I} / Z(\Lambda)=\prod_{s \in I} \operatorname{PGL}_{2}\left(\mathbb{F}_{q_{s}}\right) \times$ 
$\prod_{s \notin I} \mathbb{F}_{q_{s}}^{\times}$. According to Tits' amalgam theorem [T1, §14], the group $\Lambda / Z(\Lambda)$ is the limit of the inductive system of the groups $L_{I} / Z(\Lambda) \ltimes U^{I}=P^{I} / Z(\Lambda)$ indexed by the finite spherical subsets $I$.

For any prime power $q, \mathrm{GL}_{2}\left(\mathbb{F}_{q}\right)$ contains the multiplicative group of the field $\mathbb{F}_{q^{2}}$, a cyclic subgroup of order $q^{2}-1$, and hence $\mathrm{PGL}_{2}\left(\mathbb{F}_{q}\right)$ contains a cyclic subgroup of order $q+1$. Since the first group is transitive on the projective line, so is the second. Therefore we can see $\mathbb{Z} /(q+1) \mathbb{Z}$ as a subgroup of $\mathrm{PGL}_{2}\left(\mathbb{F}_{q}\right)$ which acts simply transitively on the chambers of the corresponding building $\mathbb{P}^{1}\left(\mathbb{F}_{q}\right)[\mathrm{Ch}$, Proposition 5]. For each spherical subset $I$, this remark gives the first inclusion in the chain $\prod_{s \in I} \mathbb{Z} /\left(q_{s}+1\right) \mathbb{Z}<L_{I} / Z(\Lambda)<P_{I} / Z(\Lambda)$. Forgetting the middle term we obtain a morphism of inductive systems, from the one defining $\Gamma_{(W, S), 1+q}$ to the one defining $\Lambda / Z(\Lambda)$. We obtain the desired inclusion by taking the corresponding direct limits.

Finally, we have coexistence of lattices linear in characteristic 0 and non-linear lattices in the same topological group of root datum type whenever the uniform lattice $\Gamma_{(W, S), 1+q}$ is known to be linear. This is the case for the lattices considered in [Bou], where the Weyl group is right-angled Fuchsian (see the example in 3.A).

Corollary. Let $(W, S)$ be the right-angled Coxeter system corresponding to the tiling of the hyperbolic plane $\mathbb{H}^{2}$ by regular right-angled $r$-gons. Let $\left\{1+q_{s}\right\}_{s \in S}$ be a parameter system consisting of cardinalities of finite projective lines, with $q_{s}$ prime to $q_{t}$ for some $s \neq t$. Let $\Lambda$ be defined as above and let $G$ be the topological group attached to $\Lambda$. If $q=\min _{s \in S} q_{s}$ is large enough, then $G$ contains non-linear non-uniform lattices, whereas all its uniform lattices are linear in characteristic 0.

Proof. The assertion on non-linear lattices follows from the corollary in 4.A, and it follows from [Hag, Introduction] that any uniform lattice in a right-angled Fuchsian building is linear in characteristic 0 .

Remark. If $\Gamma$ is a lattice in a higher-rank non-archimedean simple Lie group $G$, and if $X$ is a proper CAT $(-1)$-space with cocompact isometry group, then any $\Gamma$-action on $X$ has a global fixed point [BM, Corollary 0.5]. The coexistence phenomenon of the above corollary is thus excluded in the classical algebraic situation, unless the building of $G$ is a tree (i.e. $G$ is a rank one group). 


\section{References}

[A] P. Abramenko, Twin buildings and applications to S-arithmetic groups. Lecture Notes in Math. 1641, Springer-Verlag, Berlin 1996. Zbl 0908.20003 MR 1624276

[BM] M. Burger, S. Mozes, CAT $(-1)$-spaces, divergence groups and their commensurators. J. Amer. Math. Soc. 9 (1996), 57-93. Zbl 0847.22004 MR 1325797

[Bou] M. Bourdon, Immeubles hyperboliques, dimension conforme et rigidité de Mostow. Geom. Funct. Anal. 7 (1997), 245-268. Zbl 0876.53020 MR 1445387

[BS] U. Bader, Y. Shalom, Factor and normal subgroup theorems for lattices in product of groups. To appear in Invent. Math.

[CG] L. Carbone, H. Garland, Existence of lattices in Kac-Moody groups over finite fields. Commun. Contemp. Math. 5 (2003), 813-867. Zbl 02078564 MR 2017720

[Ch] F. Choucroun, Sous-groupes discrets des groupes $p$-adiques de rang un et arbres de Bruhat-Tits. Israel J. Math. 93 (1996), 195-219. Zbl 0844.05051 MR 1380643

[Cho] A. Chosson, Isométries dans les immeubles jumelés et construction d'automorphismes de groupes de Kac-Moody. PhD Thesis, Université Amiens, 2000.

[D] M. Davis, Buildings are CAT(0). In Geometry and Cohomology in Group Theory (P. H. Kropholler, G. A. Niblo, R. Stöhr, eds.), London Math. Soc. Lecture Note Ser. 252, Cambridge University Press, Cambridge 1997, 108-123. Zbl 0978.51005 MR 1709955

[GP] D. Gaboriau, F. Paulin, Sur les immeubles hyperboliques. Geom. Dedicata 88 (2001), 153-197. Zbl 1020.51007 MR 1877215

[Hag] F. Haglund, Commensurability and separability of quasiconvex subgroups. Preprint, Université Paris Sud Orsay, 2005.

[Hée] J.-Y. Hée, Construction de groupes tordus en théorie de Kac-Moody. C. R. Acad. Sci. Paris Ser. I Math. 310 (1990), 77-80. Zbl 0707.22008 MR 1044619

[HP] F. Haglund, F. Paulin, Constructions arborescentes d'immeubles. Math. Ann. 325 (2003), 137-164. Zbl 1025.51014 MR 1957268

[KP] V. Kac, D. Peterson, Defining relations for certain infinite-dimensional groups. Astérisque No. Hors-Sér. 1985, 165-208. Zbl 0625.22014 MR 0837201

[Mar] G. A. Margulis, Discrete subgroups of semisimple Lie groups. Ergeb. Math. Grenzgeb. (3) 17, Springer-Verlag, Berlin 1991. Zbl 0732.22008 MR 1090825

[Mou] G. Moussong, Hyperbolic Coxeter groups. PhD Thesis, Ohio State University, 1988.

[Ré1] B. Rémy, Groupes de Kac-Moody déployés et presque déployés. Astérisque 277, Soc. Math. France, 2002. Zbl 1001.22018 MR 1909671

[Ré2] B. Rémy, Construction de réseaux en théorie de Kac-Moody. C. R. Acad. Sc. Paris Sér. I Math. 329 (1999), 475-478. Zbl 0933.22029 MR 1715140

[Ré3] B. Rémy, Classical and non-linearity properties of Kac-Moody lattices. In Rigidity in Dynamics and Geometry (Newton Institute 2000, M. Burger and A. Iozzi, eds.), SpringerVerlag, Berlin 2002, 391-406. Zbl 1029.22028 MR 1919413

[Ré4] B. Rémy, Kac-Moody groups: split and relative theories. Lattices. In Groups: Topological, Combinatorial and Arithmetic Aspects (T. W. Müller, ed.), London Math. Soc. Lecture Note Ser. 311, Cambridge University Press, Cambridge 2004, 487-541. Zbl 02152962 MR 2073357 
[Ré5] B. Rémy, Topological simplicity, commensurator superrigidity and non-linearity of KacMoody groups. Appendix by Patrick Bonvin: Strong boundaries and commensurator superrigidity, Geom. Funct. Anal. 14 (2004), 810-852. Zbl 1056.22015 MR 2084981

[Ro1] M. Ronan, Lectures on buildings. Perspect. Math. 7, Academic Press, Boston, MA, 1989. Zbl 0694.51001 MR 1005533

[Ro2] M. Ronan, Local isometries of twin buildings. Math. Z. 234 (2000), 435-455. Zbl 01502084 MR 1774092

[S] J.-P. Serre, Arbres, amalgames, SL2. Astérisque 46, Soc. Math. France, 1977. Zbl 0369.20013 MR 0476875

[T1] J. Tits, Ensembles ordonnés, immeubles et sommes amalgamées. Bull. Soc. Math. Belg. Sér. A 38 (1986), 367-387. Zbl 0626.20027 MR 0885545

[T2] J. Tits, Uniqueness and presentation of Kac-Moody groups over fields. J. Algebra 105 (1987), 542-573. Zbl 0626.22013 MR 0873684

[T3] J. Tits, Théorie des groupes. Résumé de cours, Ann. Collège France 89 (1988/89), 81-95. MR 1320796

[T4] J. Tits, Théorie des groupes. Résumé de cours, Ann. Collège France 90 (1989/90), 87-103. MR 1320801

[T5] J. Tits, Twin buildings and groups of Kac-Moody type. In Groups, Combinatorics \& Geometry (M. Liebeck and J. Saxl, eds.), London Math. Soc. Lecture Note Ser. 165, Cambridge University Press, Cambridge 1992, 249-286. Zbl 0851.22023 MR 1200265

Received May 3, 2002; revised May 9, 2005

Bertrand Rémy, Institut Camille Jordan, UMR 5208 CNRS/Université Lyon 1, 21 avenue Claude Bernard, 69622 Villeurbanne cedex, France

E-mail: remy@math.univ-lyon1.fr

Mark Ronan, Department of Mathematics, Statistics and Computer Science, University of Illinois at Chicago, 851 S. Morgan Street, Chicago, IL 60607-7045, U.S.A.

E-mail: ronan@uic.edu 